\title{
Une théorie du corps de classes local non abélien
}

\author{
François Laubie
}

\begin{abstract}
A non-Abelian generalization of local class field theory, which can describe non-abelian Galois groups in terms of the ground field, is given. The absolute Galois group of a local field is canonically defined up to an inner automorphism and the natural approach of describing class functions on it leads to Langland's philosophy. Another way to describe the absolute Galois group is to rigidify its structure by means of an arbitrary lifting of the Frobenius automorphism. This point of view is used in the metabelian local class field theory of Koch and de Shalit and its generalization by Gurevich. Iterating a slightly modified version of metabelian local class field theory on some fields of norms leads to a 'non-abelian local class field theory'.
\end{abstract}

\section{Introduction}

Soit $K$ un corps local à corps résiduel fini $\mathbb{F}_{q}$. La théorie du corps de classes consiste à décrire les extensions abéliennes de $K$ et leur ramification en termes du corps de base $K$. Le pivot de la théorie est l'application de réciprocité d'Artin qui induit un isomorphisme entre le complété profini $\widehat{K^{\times}}$du groupe multiplicatif $K^{\times}$de $K$ et le groupe de Galois $\operatorname{Gal}\left(K_{a b} / K\right)$ de l'extension abélienne maximale de $K$.

Zink a prolongé cet isomorphisme en un isomorphisme entre une extension de $\widehat{K^{\times}}$par son carré alterné $\Lambda^{2}\left(\widehat{K^{\times}}\right)$et le groupe de Galois de l'extension nilpotente de classe 2 maximale de $K$ (voir [Zin83]).

Une telle généralisation de la théorie du corps de classes local ne peut pas être menée à bien jusqu'au bout, c'est à dire jusqu'à la description du groupe de Galois absolu $G_{K}=\operatorname{Gal}\left(K_{\text {sep }} / K\right)$ en termes d'objets issus exclusivement du corps de base $K$, parce que $G_{K}$ n'est canoniquement défini qu'à un automorphisme intérieur près.

Les 'principes de fonctorialité' de Langlands ont permis de contourner cet obstacle et le théorème de Harris-Taylor [HT01] est une généralisation non abélienne de la théorie du corps de classes local. Il y en a d'autres qu'il n'y a pas de raison d'ignorer.

Dans la théorie des 'micropremiers' de Neukirch [Neu94], il y a l'idée très naturelle de décrire l'ensemble des classes de conjugaison de $G_{K}$ à partir du corps de base. La théorie du corps de classes local métabélien de Koch et de Shalit [KdS96] consiste à décrire un isomorphisme de réciprocité pour le groupe de Galois de l'extension métabélienne maximale de $K$ dépendant d'une donnée additionnelle qu'ils appellent un dévissage de Lubin-Tate (a Lubin-Tate splitting) et qui peut être vu comme un micropremier de degré 1 au sens de Neukirch.

À l'aide de la théorie du corps des normes de Fontaine et Wintenberger [Win83], Gurevich a proposé une généralisation de cette théorie aux extensions galoisiennes résolubles de classe finie

Received 12 May 2005, accepted in final form 2 August 2006.

2000 Mathematics Subject Classification 11S31 (primary), 11S37 (secondary).

Keywords: local class field theory, Lubin-Tate formal groups, fields of norms theory.

This journal is (C) Foundation Compositio Mathematica 2007. 


\section{F. LAUBiE}

maximales de $K$ (voir [Gur98]). Fesenko a également étudié une application de réciprocité non abélienne qui généralise celle de Koch et de Shalit [Fes01].

Dans cet article, on construit une version légèrement modifiée de l'application de réciprocité de Koch et de Shalit, qui s'applique itérativement à une suite de corps de normes issus de la théorie de Fontaine et Wintenberger. Il en résulte une description récursive du groupe de Galois absolu $G_{K}$. Cette description n'est pas complète faute d'avoir pris en compte la ramification. Elle fournit néanmoins des généralisations naturelles à la plupart des théorèmes principaux de la théorie classique du corps de classes local. Koch et de Shalit ont formulé dans [KdS96] 'certains de leurs théorèmes dans un cadre plus général que nécessaire' parce qu'ils prévoyaient que 'leurs méthodes seraient généralisées au-delà du cas métabélien'. C'est ce qui est fait dans cet article.

Dans toute la suite, un dévissage de Lubin-Tate sur $K$ est fixé ; il s'agit d'un automorphisme de Frobenius $\phi \in G_{K}$ qui détermine une section de la suite exacte

$$
\begin{aligned}
0 \longrightarrow G_{K}^{0} \longrightarrow G_{K} \longrightarrow \widehat{\mathbb{Z}} \longrightarrow 0 \\
\phi \mapsto 1
\end{aligned}
$$

où $G_{K}^{0}=\operatorname{Gal}\left(K_{s e p} / K_{n r}\right)$ est le sous-groupe d'inertie ; la restriction de $\phi$ à $K_{n r}$ est l'automorphisme de Frobenius dans $\operatorname{Gal}\left(K_{n r} / K\right)$.

Le but de l'article est de construire un groupe complet $\mathfrak{G}(K, \phi)$ à partir de $K$ et de $\phi$ et un isomorphisme continu de $\mathfrak{G}(K, \phi)$ sur $G_{K}$ possédant les propriétés attendues d'une application de réciprocité. Les questions liées à la ramification s'avèrent délicates ; elles ne sont pas abordées ici. La stratégie se décompose en trois étapes:

(1) donner une description du groupe de Galois absolu $G_{K}$ de $K$ en termes de séries formelles à coefficients dans des corps finis provenant de la théorie du corps des normes et de la notion d'étiquetage de Lubin-Tate due à Koch et de Shalit [KdS96] ;

(2) interpréter ces séries formelles comme des isomorphismes de certains groupes formels grâce à la théorie des groupes et des applications de réciprocité de Koch et de Shalit [KdS96] ; l'analogue de l'application norme dans les groupes de Koch-de Shalit permet d'en faire un système projectif compatible avec les applications de réciprocité et de définir ainsi un groupe de Koch-de Shalit absolu isomorphe à $G_{K}$;

(3) décrire récursivement à partir du corps $K$, en termes de séries formelles, ce groupe de Koch-de Shalit absolu et l'application de réciprocité, grâce au choix d'une famille convenable d'extensions compatibles et arithmétiquement profinies de $K$ qui recouvrent $K_{\text {sep }}$, qui ne dépend que de $K$ et de $\phi$. Cela donne, pour tout entier $d \geqslant 1$, une description du groupe de Galois $\mathcal{G}_{d}(K, \phi)$ de l'extension galoisienne maximale de $K$ fixe par $\phi^{d}$ et la description de $G_{K}=\lim _{d} \mathcal{G}_{d}(K, \phi)$ en résulte.

Dans la section 1, nous donnons les grandes lignes de la construction de l'application générale de réciprocité en ne précisant que les détails strictement nécessaires sur les outils utilisés. C'est ainsi que, pour tout ce qui concerne la théorie du corps des normes, le lecteur est renvoyé à l'article [Win83] bien que, dans tout ce qui suit, cette théorie soit cruciale ; elle permet notamment de se passer des séries de Coleman utilisées par Koch et de Shalit [KdS96]. La section 2 est le développement de la section 1 : elle en contient les démonstrations ; on établit aussi que les théorie du corps de classes local et du corps des normes commutent. La section 3 est consacrée à l'énoncé détaillé d'un théorème du corps de classes local non abélien sur le modèle du corps de classes local abélien classique, ce qui nécessite de décrire auparavant le groupe de Koch-de Shalit absolu en termes de séries formelles. Nous terminons en signalant les liens entre cette théorie, celle de Gurevich et celle de Fesenko. 


\section{UNE THÉORIE DU CORPS DE CLASSES LOCAL NON ABÉLIEN}

\section{Résumé de la construction}

On rappelle que $\phi$ est un dévissage de Lubin-Tate sur $K$ fixé une fois pour toutes.

\section{1 Étiquettes}

Pour toute extension $L \subset K_{\text {sep }}$ de $K$, on désigne par $\widetilde{L}$ le complété de l'extension maximale non ramifiée $L_{n r}$ de $L$ contenu dans un complété fixé $\widetilde{K_{\text {sep }}}$ de $K_{\text {sep }}$.

Une extension séparable $L / K$ est dite compatible si elle est de degré résiduel $f$ fini et si elle est fixe par $\phi^{f}$; si, de plus $L / K$ est finie, $\phi_{L}=\phi^{f}$ est un dévissage de Lubin-Tate sur $L$ et ce sera le seul qu'on considèrera.

Il existe une unique famille d'uniformisantes $\left\{\varpi_{L} \in \widetilde{L}\right\}, L$ parcourant les sous-extensions finies de $K_{\text {sep }} / K$, telle que $N_{\widetilde{M} / \widetilde{L}}\left(\varpi_{M}\right)=\varpi_{L}$ si $\widetilde{M} \supset \widetilde{L}$ et que $\varpi_{L} \in L$ si $L$ est compatible. On dit alors que $\varpi_{L}$ est l'étiquette de $L$.

Il s'agit, en fait, du cas particulier le plus simple de la définition originale des étiquettes donnée par Koch et de Shalit dans [KdS96].

Si $L / K$ est une extension séparable infinie compatible, on définit l'étiquette $\varpi_{L}$ de $L$ comme la famille d'étiquettes $\left\{\varpi_{E}\right\}$ où $E / K$ parcourt les sous-extensions finies de $L / K$ telles que $L / E$ soit totalement ramifiée.

Si, en outre, $L$ est une extension arithmétiquement profinie de $K$, son étiquette $\varpi_{L}$ est une uniformisante de son corps des normes.

Désormais la notation $\varpi_{L}$ est réservée aux étiquettes.

Un dévissage de Lubin-Tate détermine aussi canoniquement une famille de groupes formels de Lubin-Tate : si l'extension $L / K$ est finie et compatible, il existe un unique groupe formel de LubinTate sur $L$ associé à l'uniformisante $\varpi_{L}$ de $L$ dont le module de Tate est engendré par $\varpi_{L_{a b}}$ (voir [KdS96]). On dit que c'est le groupe étiqueté de $L$ et on le note $F_{L}$.

Dans tout ce qui suit, les seuls groupes formels que l'on considère, sont des groupes formels de Lubin-Tate ; en outre, si $F$ est un groupe formel de Lubin-Tate associé à une uniformisante $\pi$, les seuls endomorphismes de $F$ que l'on considère sont ceux qui commutent avec la série $[\pi](X) \equiv$ $\pi X \bmod X^{2}$ qui caractérise $F \operatorname{par}[\pi] \in \operatorname{End}(F)$; cette précision n'est utile qu' en caractéristique non nulle.

\subsection{Corps de normes (voir [Win83])}

Tous les plongements de corps valués que l'on considère sont supposés continus.

Pour toute extension de corps $E / F$ et tout groupe d'automorphismes $\mathcal{G}$ de $F$, on note Aut $\mathcal{G}$ $(E / F)=\left\{\sigma \in \operatorname{Aut}(E) ; \sigma(K)=K,\left.\sigma\right|_{K} \in \mathcal{G}\right\}$.

Étant donnée une extension galoisienne arithmétiquement profinie $L$ de $K$ de groupe de Galois $\mathcal{G}$, son corps des normes $\mathfrak{N}_{K}(L)$ est canoniquement muni d'un groupe d'automorphismes $\mathfrak{N}_{K}(\mathcal{G})$ isomorphe à $\mathcal{G}$; il y a une équivalence de catégories notée $\mathfrak{N}_{L / K}$ entre la catégorie des extensions séparables de $L$ et des $L$-plongements, et celle des extensions séparables du corps des normes $\mathfrak{N}_{K}(L)$ de $L / K$ et des $\mathfrak{N}_{K}(L)$-plongements, induisant un isomorphisme,

$$
\mathfrak{N}_{L / K}: \operatorname{Aut}_{\mathcal{G}}(M / L) \longrightarrow \operatorname{Aut}_{\mathfrak{N}_{K}(\mathcal{G})}\left(\mathfrak{N}_{L / K}(M) / \mathfrak{N}_{K}(L)\right),
$$

pour toute extension séparable $M$ de $L$ (pour $M=L$, c'est l'isomorphisme déjà cité de $\mathcal{G}$ sur $\left.\mathfrak{N}_{K}(\mathcal{G})\right)$.

Cet isomorphisme est, de façon naturelle, compatible avec la ramification. L'extension $\mathfrak{N}_{L / K}(M)$ du corps local $\mathfrak{N}_{K}(L)$ est arithmétiquement profinie si et seulement si $M / K$ l'est et dans ce cas, $\mathfrak{N}_{\mathfrak{N}_{K}(L)}\left(\mathfrak{N}_{L / K}(M)\right)=\mathfrak{N}_{K}(M)$ et, pour tout $K$-automorphisme $\sigma$ de $K_{\text {sep }}$ tel que $\sigma(L)=L$ 


\section{F. LAUBiE}

et $\sigma(M)=M, \mathfrak{N}_{\mathfrak{N}_{L / K}(M) / \mathfrak{N}_{K}(L)}\left(\mathfrak{N}_{L / K}(\sigma)\right)=\mathfrak{N}_{M / K}(\sigma)$. C'est ce que l'on appelle les propriétés de transitivité des corps de normes.

On peut effectuer les identifications suivantes :

$$
\begin{gathered}
\mathfrak{N}_{L / K}\left(K_{\text {sep }}\right)=\mathfrak{N}_{K}(L)_{\text {sep }}, \\
\mathfrak{N}_{L / K}\left(K_{n r} L\right)=\mathfrak{N}_{K}(L)_{n r}, \\
\mathfrak{N}_{\widetilde{K}}(\widetilde{K} L)=\widetilde{\mathfrak{N}_{K}(L)} \subset \widehat{\mathfrak{N}_{K}(L)_{\text {sep }}} .
\end{gathered}
$$

Si, de plus $L / K$ est compatible, $\mathfrak{N}_{L / K}\left(\phi_{L}\right)$ est un dévissage de Lubin-Tate sur $\mathfrak{N}_{K}(L)$ et le foncteur $\mathfrak{N}_{L / K}$ applique les extensions compatibles de $L$ sur les extensions compatibles sur $\mathfrak{N}_{K}(L)$ en conservant les mêmes étiquettes ; donc $\varpi_{L}=\varpi_{\mathfrak{N}_{K}(L)}$ et nous pouvons autoriser l'abus de langage :

$$
\mathfrak{N}_{L / K}\left(\phi_{L}\right)=\phi_{L}
$$

\subsection{Projections canoniques des corps de normes}

Soit $L / K$ une extension arithmétiquement profinie et compatible et soit $\mathbb{F}_{q_{L}}$ son corps résiduel. On peut identifier les extensions de corps $\mathfrak{N}_{\widetilde{K}}(\widetilde{K} L) / \mathfrak{N}_{K}(L)$ et $\overline{\mathbb{F}}_{q}\left(\left(\varpi_{L}\right)\right) / \mathbb{F}_{q_{L}}\left(\left(\varpi_{L}\right)\right.$ ) (sauf dans le cas où car $(K)=0$ et où l'extension $L / K$ est finie, auquel cas l'identification est purement ensembliste).

Le corps résiduel $\overline{\mathbb{F}}_{q}$ de $\widetilde{K} L$ se plonge dans le corps de normes $\mathfrak{N}_{\widetilde{K}}(\widetilde{K} L)$ de la façon suivante : soit $\xi$ appartenant au corps résiduel de $\widetilde{K} L$ et soit $x$ le représentant de Teichmuller de $\xi$ dans la sous-extension modérément ramifiée maximale $\mathcal{M} / \widetilde{K}$ de $\widetilde{K} L / \widetilde{K}$ alors la suite des $x^{[E: \mathcal{M}]^{-1}}, E$ parcourant les sous-extensions finies de $\widetilde{K} L / \mathcal{M}$, définit un élément $\bar{\xi}$ de $\mathfrak{N}_{\widetilde{K}}(\widetilde{K} L)$ et $\xi \mapsto \widehat{\xi}$ est le plongement retenu; le plongement du corps résiduel $\mathbb{F}_{q_{L}}$ de $L$ dans $\mathfrak{N}_{K}(L)$ est défini de manière analogue.

Pour tout entier $d \geqslant 1$, on note $K_{d}$ l'extension non ramifiée de $K$ de degré $d$ et, pour toute extension séparable $E$ de $K$, on note $E_{d}=K_{d} E$.

Étant données deux extensions arithmétiquement profinies $M$ et $L$ de $K$ ou de $\widetilde{K}$ telles que $L \subset M$, on a, par définition, $\mathfrak{N}_{\widetilde{K}}(\widetilde{K} L)^{\times}=\lim _{\longleftarrow} E^{\times}$(respectivement $\left.\mathfrak{N}_{\widetilde{K}}(\widetilde{K} M)^{\times}=\lim _{\longleftarrow} E^{\times}\right)$les limites projectives étant définies par les normes entre les sous-extensions finies $E / \widetilde{K}$ de $\widetilde{K} L / \widetilde{K}$ (respectivement de $\widetilde{K} M / \widetilde{K})$. Le système projectif définissant $\mathfrak{N}_{\widetilde{K}}(\widetilde{K} L)^{\times}$étant un sous-système de celui qui définit $\mathfrak{N}_{\widetilde{K}}(\widetilde{K} M)^{\times}$, il en résulte un homomorphisme de groupes canonique (dit projection canonique) :

$$
\widetilde{N}_{M / L}: \mathfrak{N}_{\widetilde{K}}(\widetilde{K} M)^{\times} \longrightarrow \mathfrak{N}_{\widetilde{K}}(\widetilde{K} L)^{\times}
$$

qui applique $\mathfrak{N}_{K}(M)^{\times}$dans $\mathfrak{N}_{K}\left(L_{d}\right)^{\times}$où $d$ est le degré résiduel de $M / K$, et qui coïncide avec la norme $N_{\widetilde{K} M / \widetilde{K} L}$ quand l'extension $M / L$ est finie.

Si l'image canonique (par $\left.\widetilde{N}_{M / L}\right)$ de

$$
\sum_{i} \beta_{i} \varpi_{M}^{i} \in \overline{\mathbb{F}}_{q}\left(\left(\varpi_{M}\right)\right)=\mathfrak{N}_{\widetilde{K}}(\widetilde{K} M)
$$

est

$$
\sum_{i} \alpha_{i} \varpi_{L}^{i} \in \overline{\mathbb{F}}_{q}\left(\left(\varpi_{L}\right)\right)=\mathfrak{N}_{\widetilde{K}}(\widetilde{K} L)
$$

on note

$$
\vartheta_{M / L}\left(\sum_{i} \beta_{i} X^{i}\right)=\sum_{i} \alpha_{i} X^{i},
$$

$\vartheta_{M / L}$ est un endomorphisme du groupe multiplicatif de $\overline{\mathbb{F}}_{q}((X))^{\times}$, sauf dans le cas où car $(K)=0$ et où l'extension $L / K$ est finie. À cause des propriétés de transitivité des corps de normes, les notations 


\section{UNE THÉORIE DU CORPS DE CLASSES LOCAL NON ABÉLIEN}

$\widetilde{N}_{M / L}$ et $\vartheta_{M / L}$ gardent un sens évident quand $M$ est une extension arithmétiquement profinie d'un corps de normes de la forme $\mathfrak{N}_{K}(L)$ ou $\mathfrak{N}_{\widetilde{K}}(\widetilde{K} L)$.

Il est parfois plus approprié d'indexer les projections canoniques avec les corps de normes : $\widetilde{N}_{\mathfrak{N}_{K}(M) / \mathfrak{N}_{K}(L)}=\widetilde{N}_{M / L}$, ce qui, compte-tenu des propriétés de transitivité des corps de normes, ne présente aucun inconvénient.

\subsection{Description des groupes de Galois en termes de séries formelles : les groupes $\mathcal{G}_{L}(K, \phi)$}

Supposons de plus que $M$ et $L$ soient des extensions infinies et compatibles de $K$ telles que $K_{n r} L / K$ et $K_{n r} M / K$ soient galoisiennes avec $L \subset M$.

Soit $\sigma$ un $K$-automorphisme de $K_{\text {sep }}$ tel que $\sigma_{\left.\right|_{n r}}=\phi^{\nu}$. La restriction à $\mathfrak{N}_{\widetilde{K}}(\widetilde{K} M)$ de $\mathfrak{N}_{\widetilde{K} M / \widetilde{K}}(\sigma)$ est un automorphisme de $\mathfrak{N}_{\widetilde{K}}(\widetilde{K} M)=\overline{\mathbb{F}}_{q}\left(\left(\varpi_{M}\right)\right)$ déterminé par le couple

$$
\left(\nu, \sigma_{M}(X)\right) \in \widehat{\mathbb{Z}} \times X \overline{\mathbb{F}}_{q}[[X]]
$$

tel que

$$
\mathfrak{N}_{\widetilde{K} M / \widetilde{K}}(\sigma)\left(\sum_{i} \alpha_{i} \varpi_{M}^{i}\right)=\sum_{i} \phi^{\nu}\left(\alpha_{i}\right) \sigma_{M}\left(\varpi_{M}\right)^{i}
$$

pour tout $\sum_{i} \alpha_{i} \varpi_{M}^{i} \in \overline{\mathbb{F}}_{q}\left(\left(\varpi_{M}\right)\right)$.

$\operatorname{Ainsi} \operatorname{Gal}\left(K_{n r} M / K\right)$ s'identifie à un groupe, noté $\widetilde{\mathcal{G}}_{M}(K, \phi)$, formé de couples $\left(\nu, \sigma_{M}(X)\right) \in$ $\widehat{\mathbb{Z}} \times X \overline{\mathbb{F}}_{q}[[X]]$ pour la loi :

$$
\left(\mu, \tau_{M}(X)\right)\left(\nu, \sigma_{M}(X)\right)=\left(\mu+\nu, \phi^{\mu} \sigma_{M} \circ \tau_{M}(X)\right)
$$

où $\phi^{\mu} \sigma_{M}$ désigne l'action coefficient par coefficient de $\phi^{\mu}$ sur la série $\sigma_{M}$.

Supposons, de plus, que l'extension $M / K$ (respectivement $L / K$ ) soit galoisienne de degré résiduel $d_{M}$ (respectivement $\left.d_{L}\right)$. Les séries $\sigma_{M}(X)$ sont alors à coefficients dans le corps fini $\mathbb{F}_{q^{d}}$ et $\widetilde{\mathcal{G}}_{M}(K, \phi)$ se projette sur

$$
\mathcal{G}_{M}(K, \phi)=\left\{\left(\bar{\nu}, \sigma_{M}(X)\right) \in \mathbb{Z} / d_{M} \mathbb{Z} \times X \mathbb{F}_{q^{d_{M}}}[[X]] ;\left(\nu, \sigma_{M}(X)\right) \in \widetilde{\mathcal{G}}_{M}(K, \phi)\right\}
$$

qui s'identifie à $\operatorname{Gal}(M / K)$ et le diagramme suivant commute.

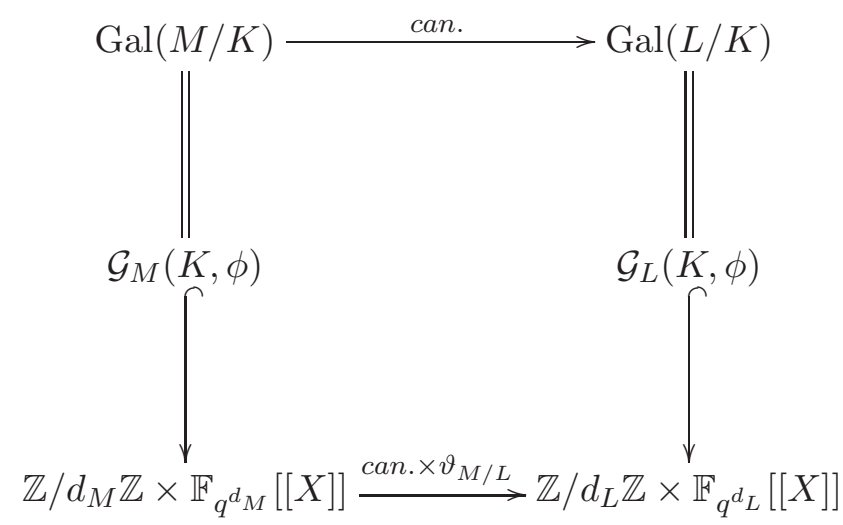

Ainsi le groupe de Galois absolu $G_{K}$ s'identifie au groupe

$$
\mathcal{G}(K, \phi)=\lim _{L} \mathcal{G}_{L}(K, \phi),
$$

$L$ parcourant n'importe quel ensemble d'extensions galoisiennes, compatibles, infinies et arithmétiquement profinies de $K$ qui recouvrent $K_{\text {sep }}$. 


\section{F. LAUBiE}

\subsection{Groupes de Koch-de Shalit}

Pour tout corps discrètement valué $E, U(E)$ désigne le groupe des unités de $E$ et $\left(U^{i}(E)\right)$ sa filtration usuelle.

Soit $L$ le corps des normes $\mathfrak{N}_{K}(\mathcal{L})$ d'une extension galoisienne, compatible $\mathcal{L} / K$; soient $\mathfrak{g}_{L}=$ $\mathfrak{N}_{K}(\operatorname{Gal}(\mathcal{L} / K)), \Gamma=\operatorname{Aut}_{\mathfrak{g}_{L}}\left(L_{n r} / L\right), G=\operatorname{Aut}_{\mathfrak{g}_{L}}\left(L_{a b} / L\right), H=\operatorname{Gal}\left(L_{a b} / L_{n r}\right)$ et $d_{L}$ le degré résiduel de $\mathcal{L} / K$. Ces hypothèses contiennent le cas où $\mathcal{L} / K$ est une extension finie, c'est à dire où $L=\mathcal{L}$, $\mathfrak{g}_{L}=\operatorname{Gal}(L / K), \Gamma=\operatorname{Gal}\left(L_{n r} / K\right), G=\operatorname{Gal}\left(L_{a b} / K\right)$, etc.

Pour tout entier $i \geqslant 1$, soit $L_{i}$ le corps de classes du sous-groupe $\left\langle\varpi_{L}\right\rangle U^{i}(L)$ de $\widehat{L}^{\times}$de sorte que $L_{a b}^{\left\langle\phi_{L}\right\rangle}=\bigcup_{i} L_{i}$ est le corps de classes de $\left\langle\varpi_{L}\right\rangle$ et soit $\mathcal{L}_{i)}=\mathfrak{N}_{\mathcal{L} / K}^{-1}\left(L_{i)}\right)$; les corps $L_{a b}, L_{a b}^{\left\langle\phi_{L}\right\rangle}, \mathcal{L}_{a b}, \mathcal{L}_{a b}^{\left\langle\phi_{L}\right\rangle}=\bigcup_{i} \mathcal{L}_{i)}=\mathfrak{N}_{K}\left(L_{a b}^{\left\langle\phi_{L}\right\rangle}\right)$, ont la même étiquette $\varpi_{L_{a b}}=\left\{\varpi_{L_{i}}\right\}_{i}$.

Nous avons le diagramme suivant.

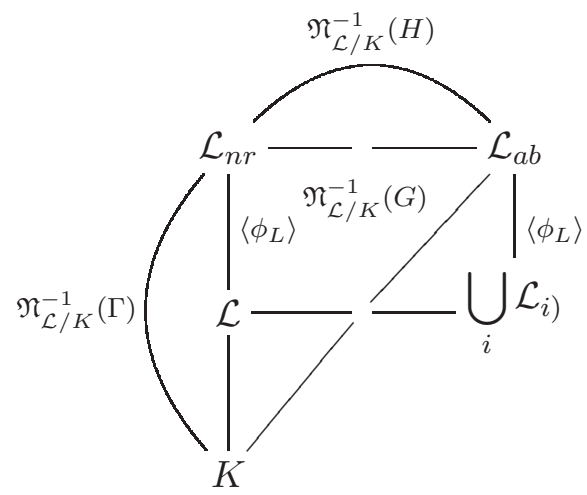

Le groupe de Koch-de Shalit $G\left(\mathfrak{g}_{L}, \varpi_{L}\right)$ est

$$
G\left(\mathfrak{g}_{L}, \varpi_{L}\right)=\left\{(\gamma, b) \in \Gamma \times U(\tilde{L}) \phi_{L}(b) / b=\gamma\left(\varpi_{L}\right) / \varpi_{L}\right\}
$$

pour la loi $\left(\gamma_{1}, b_{1}\right)\left(\gamma_{2}, b_{2}\right)=\left(\gamma_{1} \gamma_{2}, b_{1} \gamma_{1}\left(b_{2}\right)\right)$ (voir $\left.[\operatorname{KdS} 96, \S 2.2]\right)$.

Le noyau de la première projection est $\{1\} \times U(L)$, autrement dit, on a la suite exacte de groupes :

$$
1 \longrightarrow U(L) \longrightarrow G\left(\mathfrak{g}_{L}, \varpi_{L}\right) \longrightarrow \Gamma \longrightarrow 1
$$

\subsection{Application de réciprocité de Koch-de Shalit}

ThÉorème 1 (Koch et de Shalit [KdS96]). Soit $F_{L}$ est le groupe étiqueté sur $L$; pour tout $\gamma \in \Gamma$, soit $\gamma F_{L}$ l'image coefficient par coefficient de la série $F_{L} ;$ pour tout $b \in \mathcal{O}(\widetilde{L})$, soit $[b]_{F_{L}, \gamma F_{L}}(X) \in \mathcal{O}(\widetilde{L})[[X]]$ l'unique isomorphisme de groupes formels de $F_{L}$ sur $\gamma F_{L}$ défini sur $\mathcal{O}(\widetilde{L})$ tel que $[b]_{F_{L}, \gamma F_{L}}(X) \equiv b X \bmod X^{2}$ et tel que $[b]_{F_{L}, \gamma F_{L}} \circ[a]_{F}=[a]_{\gamma F} \circ[b]_{F_{L}, \gamma F_{L}}$, pour tout $a \in \mathcal{O}(L)$.

Il existe un unique isomorphisme $\iota_{L}$ de $G\left(\mathfrak{g}_{L}, \varpi_{L}\right)$ sur $G$ tel que

$$
\forall(\gamma, b) \in G\left(\mathfrak{g}_{L}, \varpi_{L}\right), \forall i \geqslant 1, \quad \iota_{L}(\gamma, b)\left(\varpi_{\left.L_{i}\right)}\right)=\phi_{L}^{1-i}[b]_{F_{L}, \gamma F_{L}}\left(\varpi_{\left.L_{i}\right)}\right)
$$

et tel que le diagramme suivant commute

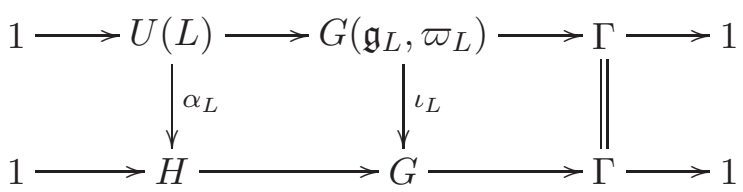

où $\alpha_{L}: u \mapsto\left(u^{-1}, L_{a b} / L\right)$ désigne l'inverse de l'application de réciprocité d'Artin. 
Soit $M=\mathfrak{N}_{L / K}(\mathcal{M})$ où $\mathcal{M} / K$ est la sous-extension galoisienne maximale de $\mathcal{L}_{a b} / K$ fixe par $\phi_{L}$ et soit

$$
\bar{G}=\operatorname{Aut}_{\mathfrak{g}_{L}}(M / L)=G / \operatorname{Gal}\left(L_{a b} / M\right)=G /\left(\left\langle\phi_{L}\right\rangle \times\left[G,\left\langle\phi_{L}\right\rangle\right]\right) .
$$

Nous avons le diagramme suivant.

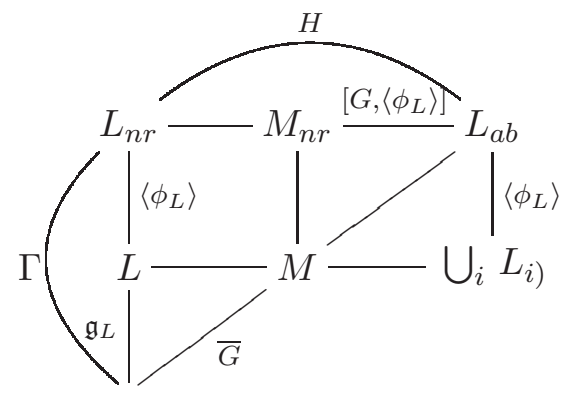

(L'absence de symbole au bas du diagramme indique que les groupes $\Gamma, \mathfrak{g}_{L}, \bar{G}$ ne sont en général pas des groupes de Galois, sauf si $[\mathcal{L}: K]<+\infty$.)

Soit $\bar{\iota}_{L}$ la restriction de l'application de réciprocité de Koch-de Shalit définie par $\forall(\gamma, b) \in$ $G\left(\mathfrak{g}_{L}, \varpi_{L}\right), \bar{\iota}_{L}(\gamma, b)=\left.\iota_{L}(\gamma, b)\right|_{M}$.

Proposition 1. Soit $V_{\mathfrak{g}_{L}}\left(\varpi_{L}\right)$ le sous-groupe fermé de $U(L)$ engendré par $\left\{g\left(\varpi_{L}\right) / \varpi_{L} ; g \in \mathfrak{g}_{L}\right\}$; $\bar{\iota}_{L}$ est un homomorphisme surjectif de $G\left(\mathfrak{g}_{L}, \varpi_{L}\right) \operatorname{sur} \bar{G}$ de noyau le sous-groupe $\left\{\phi_{L}^{\nu}\right\}_{\nu \in \widehat{\mathbb{Z}}} \times V_{\mathfrak{g}_{L}}\left(\varpi_{L}\right)$ de $G\left(\mathfrak{g}_{L}, \varpi_{L}\right)$.

\subsection{Interprétation en termes d'isomorphismes de groupes formels}

Pour tout $(\gamma, b) \in G\left(\mathfrak{g}_{L}, \varpi_{L}\right)$, soit $\nu_{\gamma} \in \widehat{\mathbb{Z}}$ tel que $\left.\gamma\right|_{K_{n r}}=\phi^{\nu_{\gamma}}$ et soit $\bar{\nu}_{\gamma}$ la classe de $\nu_{\gamma}$ modulo $d_{L}$, le degré résiduel de $\mathcal{L} / K$. On rappelle que $F_{L}$ désigne le groupe étiqueté sur $L$, que $\overline{F_{L}}$ est sa réduction modulo $\varpi_{L}$ (coefficient par coefficient) et que $\gamma F_{L}$ est l'image de $F_{L}$ par $\gamma$ (coefficient par coefficient). La réduction $\overline{[b]_{F_{L}, \gamma F_{L}}}$ modulo $\varpi_{L}$ de la série $[b]_{F_{L}, \gamma F_{L}}$ est un homomorphisme de groupes formels de $\overline{F_{L}}$ dans $\phi^{\nu_{\gamma}} \overline{F_{L}}$ à coefficients dans $\overline{\mathbb{F}_{q}}$ qui ne dépend que de $\nu_{\gamma}$ et de $b$; on note :

$$
\{b\}_{\nu_{\gamma}}=\overline{[b]_{F_{L}, \gamma F_{L}}} .
$$

La série $\vartheta_{L_{a b}^{\left\langle\phi_{L}\right\rangle} / M}\left(\{b\}_{\nu_{\gamma}}(X)\right)$ est à coefficients dans $\mathbb{F}_{q^{d} L}$ et ne dépend que de $b$ et $\bar{\nu}_{\gamma}$; on note :

$$
(b)_{\bar{\nu}_{\gamma}}(X)=\vartheta_{L_{a b}^{\left\langle\phi_{L}\right\rangle} / M}\left(\{b\}_{\nu_{\gamma}}(X)\right) .
$$

Proposition 2. Soit $(\gamma, b) \in G\left(\mathfrak{g}_{L}, \varpi_{L}\right)$ et soit $\nu_{\gamma} \in \widehat{\mathbb{Z}}$ tel que $\left.\gamma\right|_{K_{n r}}=\phi^{\nu_{\gamma}} ;$ alors :

(i) $\mathfrak{N}_{\widetilde{L} L_{a b} / \widetilde{L}}\left(\iota_{L}(\gamma, b)\right)\left(\varpi_{L_{a b}}\right)=\{b\}_{\nu_{\gamma}}\left(\varpi_{L_{a b}}\right)$; autrement dit, l'application de réciprocité de Koch-de Shalit induit un isomorphisme $(\gamma, b) \mapsto\left(\nu_{\gamma},\{b\}_{\nu_{\gamma}}(X)\right)$ de $G\left(\mathfrak{g}_{L}, \varpi_{L}\right)$ sur le quotient $\widetilde{\mathcal{G}}_{L_{a b}^{\left\langle\phi_{L}\right\rangle}}(K, \phi)$ de $\mathcal{G}(K, \phi)$.

(ii) $\mathfrak{N}_{M / L}\left(\bar{\iota}_{L}(\gamma, b)\right)\left(\varpi_{M}\right)=(b)_{\bar{\nu}_{\gamma}}\left(\varpi_{M}\right)$; autrement dit, l'application de réciprocité de Koch-de Shalit induit un homomorphisme surjectif $(\gamma, b) \mapsto\left(\bar{\nu}_{\gamma},(b)_{\bar{\nu}_{\gamma}}(X)\right)$ de $G\left(\mathfrak{g}_{L}, \varpi_{L}\right)$ sur le quotient $\mathcal{G}_{M}(K, \phi)$ de $\mathcal{G}(K, \phi)$.

On peut réitérer cette construction en partant de $\left(\mathfrak{N}_{L}(M), \mathfrak{N}_{M / L}(\bar{G})\right)$ au lieu de $\left(L, \mathfrak{g}_{L}\right)$. Pour cela on a besoin de l'analogue de la norme dans les groupes de Koch-de Shalit.

\subsection{La norme dans les groupes de Koch-de Shalit}

Soit $\mathcal{L}^{\prime}$ une extension de $K$, galoisienne, arithmétiquement profinie, compatible et contenant $\mathcal{L}$; soit $L^{\prime}=\mathfrak{N}_{K}\left(\mathcal{L}^{\prime}\right)$ et soit $\mathfrak{g}_{L^{\prime}}=\mathfrak{N}_{K}\left(\operatorname{Gal}\left(\mathfrak{L}^{\prime} / K\right)\right)$. On note $G^{\prime}, \Gamma^{\prime}, \ldots$ les objets correspondant à $G$, $\Gamma, \ldots$ associés à $L^{\prime}$ et $d=d_{L^{\prime}} / d_{L}$ le degré résiduel de $\mathcal{L}^{\prime} / \mathcal{L}$. 


\section{F. LAUBiE}

Soit

$$
\begin{gathered}
r_{L^{\prime} / L}: G\left(\mathfrak{g}_{L^{\prime}}, \varpi_{L^{\prime}}\right) \rightarrow G\left(\mathfrak{g}_{L}, \varpi_{L}\right), \\
\left(\gamma^{\prime}, b^{\prime}\right) \mapsto(\gamma, b)
\end{gathered}
$$

où $\gamma=\mathfrak{N}_{\mathcal{L} / K}\left(\left.g\right|_{\mathcal{L}_{n r}}\right), g \in \operatorname{Gal}\left(\mathcal{L}_{n r}^{\prime} / K\right)$ étant défini par $\gamma^{\prime}=\mathfrak{N}_{\mathcal{L}^{\prime} / K}(g) \in \Gamma^{\prime}$, et où $b=\prod_{0 \leqslant i<d}$ $\phi_{L}^{i}\left(\widetilde{N}_{L^{\prime} / L}\left(b^{\prime}\right)\right)$; on rappelle que $\widetilde{N}_{L^{\prime} / L}=\widetilde{N}_{\mathcal{L}^{\prime} / \mathcal{L}}$ est la projection canonique définie en $\S 1.3$.

Proposition 3. Le diagramme suivant commute.

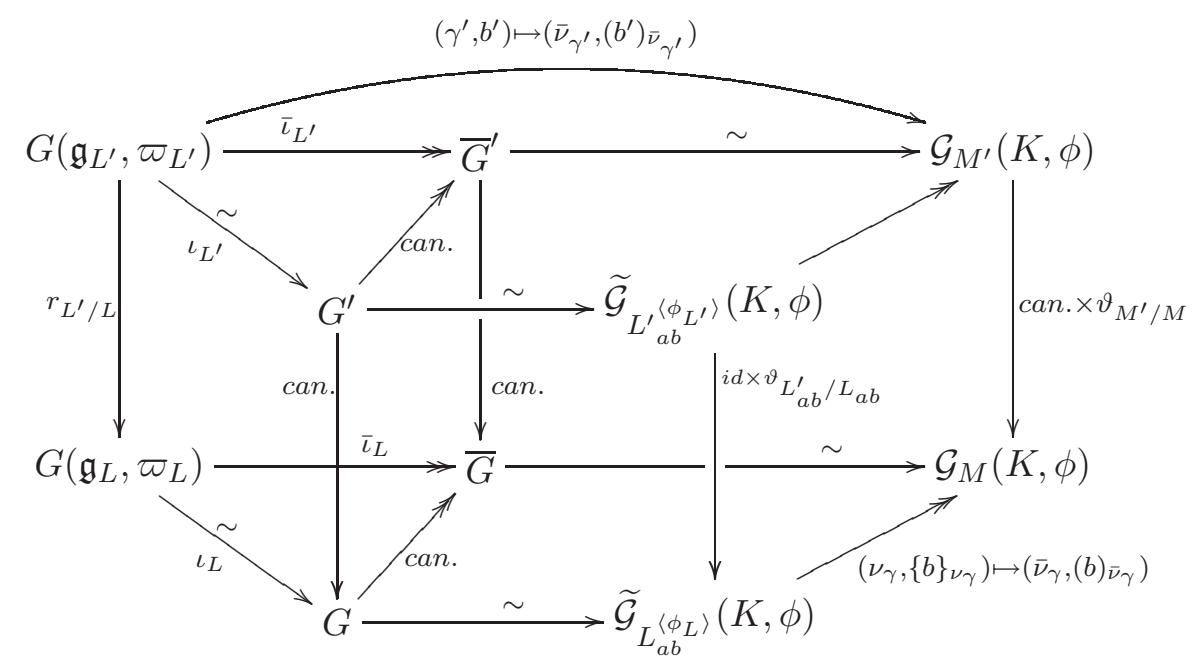

(Dans la flèche verticale de droite l'application 'can.' est la projection de $\mathbb{Z} / d_{L^{\prime}} \mathbb{Z}$ sur $\mathbb{Z} / d_{L} \mathbb{Z}$.)

Corollaire. Supposons de plus que $\mathcal{L}^{\prime} / \mathcal{L}$ soit abélienne. Soit $\gamma^{\prime} \in \Gamma^{\prime}$. Alors il existe $b^{\prime} \in U\left(\widetilde{L}^{\prime}\right)$ tel que $\phi_{L^{\prime}}\left(b^{\prime}\right) / b^{\prime}=\gamma^{\prime}\left(\varpi_{L^{\prime}}\right) / \varpi_{L^{\prime}}$ et on a $\gamma^{\prime}=\left.\iota_{L}(\gamma, b)\right|_{L_{n r}^{\prime}}$ avec $(\gamma, b)=r_{L^{\prime} / L}\left(\gamma^{\prime}, b^{\prime}\right)$, c'est à dire avec $b=\prod_{0 \leqslant i<d} \phi_{L}^{i}\left(\tilde{N}_{L^{\prime} / L}\left(b^{\prime}\right)\right)$ et $\gamma=\mathfrak{N}_{\mathcal{L} / K}\left(\left.\mathfrak{N}_{\mathcal{L}^{\prime} / K}^{-1}\left(\gamma^{\prime}\right)\right|_{\mathcal{L}_{n r}}\right)$.

\subsection{Le groupe de Koch-de Shalit absolu}

C'est la limite projective des groupes de Koch-de Shalit $G\left(\mathfrak{g}_{L}, \varpi_{L}\right)$ sous les applications $r_{L^{\prime} / L}$, $L, L^{\prime}$ parcourant les extensions galoisiennes finies compatibles de $K$ ou encore $L, L^{\prime}$ parcourant les corps de normes d'une quelconque famille d'extensions galoisiennes arithmétiquement profinies compatibles de $K$ recouvrant $K_{\text {sep }}$.

On rappelle que $K_{d}$ désigne l'extension non ramifiée de degré $d$ de $K$.

Soit $M_{d}^{(n)}$ l'extension galoisienne de $K$, résoluble de classe $n$ sur $K_{d}$, invariante par $\phi^{d}$ et maximale pour ces propriétés $\left(M_{d}^{(0)}=K_{d}\right)$, soit $\varpi_{d}^{(n)}=\varpi_{M_{d}^{(n)}}$ son étiquette et soit $\mathfrak{g}_{d}^{(n)}=$ $\mathfrak{N}_{K}\left(\operatorname{Gal}\left(M_{d}^{(n)} / K\right)\right)$.

Alors le groupe de Koch-de Shalit $G\left(\mathfrak{g}_{d}^{(n)}, \varpi_{d}^{(n)}\right)$ s'identifie à un ensemble de couples $\left(\nu, \xi_{d}^{(n)}(X)\right)$ $\in \widehat{\mathbb{Z}} \times \overline{\mathbb{F}}_{q}[[X]]$ tels que, pour tout $n^{\prime} \leqslant n$ et tout $d^{\prime} \mid d, \widetilde{N}_{M_{d}^{(n)} / M_{d^{\prime}}^{\left(n^{\prime}\right)}}\left(\xi_{d}^{(n)}\left(\varpi_{d}^{(n)}\right)\right)=\xi_{d^{\prime}}^{\left(n^{\prime}\right)}\left(\varpi_{d^{\prime}}^{\left(n^{\prime}\right)}\right)$ avec $\left(\nu, \xi_{d^{\prime}}^{\left(n^{\prime}\right)}(X)\right) \in G\left(\mathfrak{g}_{d^{\prime}}^{\left(n^{\prime}\right)}, \varpi_{d^{\prime}}^{\left(n^{\prime}\right)}\right)$. Pour $n \geqslant 1$, la loi de groupe est, (avec les notations de la Proposition 3 qui sont rappelées un peu plus loin) :

$$
\left(\nu, \xi_{d}^{(n)}\right)\left(\mu, \eta_{d}^{(n)}\right)=\left(\nu+\mu, \xi_{d}^{(n)} \cdot \phi^{\nu} \eta_{d}^{(n)} \circ\left(\xi_{d}^{(n-1)}\right)_{\bar{\nu}}\right) .
$$




\section{UNE THÉORIE DU CORPS DE CLASSES LOCAL NON ABÉLIEN}

L'application de réciprocité est :

$$
\begin{gathered}
G\left(\mathfrak{g}_{d}^{(n)}, \varpi_{d}^{(n)}\right) \stackrel{\bar{\iota}_{d}^{(n)}}{\longrightarrow} \mathcal{G}_{M_{d}^{(n+1)}}(K, \phi) \\
\left(\nu, \xi_{d}^{(n)}(X)\right) \mapsto\left(\bar{\nu},\left(\xi_{d}^{(n)}\right)_{\bar{\nu}}(X)\right)
\end{gathered}
$$

où $\bar{\nu}$ est l'image de $\nu$ modulo $d$,

$$
\left(\xi_{d}^{(n)}\right)_{\bar{\nu}}(X)=\vartheta_{\left(M_{d}^{(n)}\right)_{a b}^{\left\langle\phi^{d}\right\rangle} / M_{d}^{(n+1)}}\left(\left\{\xi_{d}^{(n)}\right\}_{\nu}(X)\right),
$$

$\left\{\xi_{d}^{(n)}\right\}_{\nu}(X)$ est l'image de la série $\left[\xi_{d}^{(n)}\left(\varpi_{d}^{(n)}\right)\right]_{F_{d}^{(n)}, \phi^{\nu} F_{d}^{(n)}}(X)$, par la réduction modulo $\varpi_{d}^{(n)}, F_{d}^{(n)}$ étant le groupe formel étiqueté du corps $\mathfrak{N}_{K}\left(M_{d}^{(n)}\right)$.

Rappellons la loi de groupe de $\mathcal{G}_{M_{d}^{(n+1)}}(K, \phi)$ donnée au $\S 1.4$ :

$$
\left(\bar{\nu},\left(\xi_{d}^{(n)}\right)_{\bar{\nu}}\right)\left(\bar{\mu},\left(\eta_{d}^{(n)}\right)_{\bar{\mu}}\right)=\left(\bar{\nu}+\bar{\mu}, \phi^{\nu}\left(\eta_{d}^{(n)}\right)_{\bar{\mu}} \circ\left(\xi_{d}^{(n)}\right)_{\bar{\nu}}\right) .
$$

Pour $n=0$, on a

$$
M_{d}^{(0)}=K_{d}, \varpi_{d}^{(0)}=\varpi_{K}, \mathfrak{g}_{d}^{(0)}=\left\{\phi^{\nu}\right\}_{\nu \in \mathbb{Z} / d \mathbb{Z}} \simeq \operatorname{Gal}\left(K_{d} / K\right),
$$

$G\left(\mathfrak{g}_{d}^{(0)}, \varpi_{d}^{(0)}\right)=\widehat{\mathbb{Z}} \times U\left(K_{d}\right)$ avec la loi de groupe $(\nu, u)(\mu, v)=\left(\nu+\mu, u \phi^{\nu}(v)\right)$, alors $G\left(\mathfrak{g}_{d}^{(0)}, \varpi_{d}^{(0)}\right) \simeq$ $\operatorname{Gal}\left(\left(K_{d}\right)_{a b} / K\right)$; l'extension abélienne de $K_{d}$ qui est corps de classes pour le sous-groupe $\left\langle\varpi_{K}\right\rangle$ de $\widehat{K_{d}^{\times}}$est en fait galoisienne sur $K$, c'est $M_{d}^{(1)}$, et, pour tout $u \in U\left(K_{d}\right), \bar{\iota}_{d}^{(0)}((0, u))=\left(u^{-1}, M_{d}^{(1)} / K_{d}\right)$ (avec $M_{d}^{(1)}=\left(K_{d}\right)_{a b}^{\left\langle\phi^{d}\right\rangle}$ ) ; pour la suite une unité $u$ de $K_{d}$ est identifiée à la série $\xi_{d}^{(0)}(X) \in \mathbb{F}_{q^{d}}[[X]]$ telle que $\xi_{d}^{(0)}\left(\varpi_{K}\right)=u$, la série $\xi_{d}^{(0)}(X)$ étant vue comme étant à coefficients dans le système de représentants multiplicatif de $\mathbb{F}_{q^{d}}$ dans $K_{d}$.

Pour $n=1$,

$$
\mathfrak{g}_{d}^{(1)}=\bar{\iota}_{d}^{(0)}\left(\mathbb{Z} / d \mathbb{Z} \times U\left(K_{d}\right)\right)=\mathcal{G}_{M_{d}^{(1)}}(K, \phi) \simeq \operatorname{Gal}\left(M_{d}^{(1)} / K\right)
$$

est constitué de couples $\left(\nu,\left\{\xi_{d}^{(0)}\right\}_{\nu}(X)\right) \in \mathbb{Z} / d \mathbb{Z} \times \mathbb{F}_{q^{d}}[[X]]$ s'identifiant aux automorphismes de $\mathfrak{N}_{K}\left(M_{d}^{(1)}\right) \simeq \mathbb{F}_{q^{d}}\left(\left(\varpi_{d}^{(1)}\right)\right)$ qui opèrent sur $\mathbb{F}_{q^{d}}$ comme $\phi^{\nu}$ et qui appliquent $\varpi_{d}^{(1)}$ sur $\left\{\xi_{d}^{(0)}\right\}_{\nu}\left(\varpi_{d}^{(1)}\right)$;

$$
\begin{gathered}
G\left(\mathfrak{g}_{d}^{(1)}, \varpi_{d}^{(1)}\right)=\left\{\left(\nu, \xi_{d}^{(1)}(X)\right) \in \widehat{\mathbb{Z}} \times \overline{\mathbb{F}}_{q}[[X]] ; \phi^{d} \xi_{d}^{(1)}(X) / \xi_{d}^{(1)}(X)=\left\{\xi_{d}^{(0)}\right\}_{\nu}(X) / X ;\right. \\
\left.\xi_{d}^{(0)}(X)=\vartheta_{M_{d}^{(1)} / K_{d}}\left(\xi_{d}^{(1)}(X)\right)\right\} \simeq \operatorname{Gal}\left(\left(M_{d}^{(1)}\right)_{a b} / K\right) .
\end{gathered}
$$

Le groupe correspondant défini et noté $\mathfrak{G}_{d}(K, \phi)$ par Koch et de Shalit, est un peu différent : c'est canoniquement un groupe quotient de $G\left(\mathfrak{g}_{d}^{(1)}, \varpi_{d}^{(1)}\right)$ isomorphe à $\operatorname{Gal}\left(\left(K_{d} M_{1}^{(1)}\right)_{a b} / K\right)$ et leur application métabélienne de réciprocité définie sur $\mathfrak{G}_{d}(K, \phi)$ est essentiellement $\bar{\iota}_{d}^{(1)}$.

Pour $n=2$,

$$
\mathfrak{g}_{d}^{(2)}=\bar{\iota}_{d}^{(1)}\left(G\left(\mathfrak{g}_{d}^{(1)}, \varpi_{d}^{(1)}\right)\right) \simeq \operatorname{Gal}\left(M_{d}^{(2)} / K\right),
$$

$\mathfrak{g}_{d}^{(2)}$ est constitué de couples $\left(\bar{\nu},\left(\xi_{d}^{(1)}\right)_{\bar{\nu}}(X)\right) \in G\left(\mathfrak{g}_{d}^{(1)}, \varpi_{d}^{(1)}\right)$ s'identifiant aux automorphismes de $\mathfrak{N}_{K}\left(M_{d}^{(2)}\right) \simeq \mathbb{F}_{q^{d}}\left(\left(\varpi_{d}^{(2)}\right)\right)$ qui opèrent sur $\mathbb{F}_{q^{d}}$ comme $\phi^{\nu}$ et qui appliquent $\varpi_{d}^{(2)}$ sur $\left(\xi_{d}^{(1)}\right)_{\bar{\nu}}\left(\varpi_{d}^{(2)}\right)$;

$$
\begin{aligned}
G\left(\mathfrak{g}_{d}^{(2)}, \varpi_{d}^{(2)}\right) & =\left\{\left(\nu, \xi_{d}^{(2)}(X)\right) \in \widehat{\mathbb{Z}} \times \overline{\mathbb{F}}_{q}[[X]] ;\right. \\
\phi^{d} \xi_{d}^{(2)}(X) / \xi_{d}^{(2)}(X) & \left.=\left(\xi_{d}^{(1)}\right)_{\bar{\nu}}(X) / X ; \xi_{d}^{(1)}(X)=\vartheta_{M_{d}^{(2)} / M_{d}^{(1)}}\left(\xi_{d}^{(2)}(X)\right)\right\} \simeq \operatorname{Gal}\left(\left(M_{d}^{(2)}\right)_{a b} / K\right) .
\end{aligned}
$$

À partir de $n=2$, la construction des $G\left(\mathfrak{g}_{d}^{(n)}, \varpi_{d}^{(n)}\right)$ est générique ; ils constituent un système projectif pour les flèches $r_{M_{d^{\prime}}^{\left(n^{\prime}\right)} / M_{d}^{(n)}},\left(d \mid d^{\prime}, n \leqslant n^{\prime}\right)$, ou si l'on préfère, pour les flèches $\vartheta_{M_{d^{\prime}}^{\left(n^{\prime}\right)} / M_{d}^{(n)}}$ 


\section{F. LAUBiE}

appliquées à la seconde coordonnée ; leur limite projective est le groupe de Koch-de Shalit absolu et l'application générale de réciprocité est la limite projective des $\bar{\iota}_{d}^{(n)}$ qui est à valeur sur $\mathcal{G}(K, \phi) \simeq$ $G_{K}$.

Notre but sera atteint lorsqu'on aura décrit récursivement à partir de $K$ et de $\phi$ le groupe $\varliminf_{\longleftarrow} G\left(\mathfrak{g}_{d}^{(n)}, \varpi_{d}^{(n)}\right)$ et l'application $\lim _{\longleftarrow} \bar{\iota}_{d}^{(n)}$.

\section{Théorie de Koch-de Shalit et corps de normes}

\section{1 Étiquetage de Lubin-Tate}

2.1.1 Étiquettes. Étant donnée une extension séparable $E$ d'un corps $F$, on note $\mathcal{F}_{E / F}$ la famille des sous-extensions finies de $E / F$.

On rappelle que $K_{d}$ désigne l'extension non ramifiée de degré $d$ de $K$.

Dorénavant on dira qu'une suite ou une famille $\left\{x_{E}\right\}_{E \in \mathcal{F}}$ indexée par $\mathcal{F} \subset \mathcal{F}_{K_{s e p} / K}$ où $x_{E}$ est élément de $E$ est projective (sous-entendu : pour les normes) si on a $N_{E^{\prime} / E}\left(x_{E^{\prime}}\right)=x_{E}$ chaque fois que $E \subset E^{\prime}$. Ainsi, si $L / K$ est une extension arithmétiquement profinie de degré résiduel $d$, une suite projective d'uniformisantes $\left(\pi_{E}\right)_{E \in \mathcal{F}_{L / K_{d}}}$ est une uniformisante du corps des normes $\mathfrak{N}_{K}(L)$.

Lemme 1 (Koch et de Shalit [KdS96, Lemma 0.2]). Il existe une unique famille projective d'uniformisantes $\left\{\varpi_{E} \in E ; E \in \mathcal{F}_{K_{\text {sep }}^{\langle\phi\rangle}}^{\langle\phi\rangle}\right\}$ indexée par $\mathcal{F}_{K_{\text {sep }}^{\langle\phi\rangle} / K} ; \varpi_{K}$ est le générateur du groupe des normes universelles de l'extension $K_{a b}^{\langle\phi\rangle} / K$.

On rappelle que $\widetilde{L}$ est le complété de l'extension maximale non ramifiée $L_{n r}$ de $L$ contenu dans un complété fixé $\widetilde{K_{\text {sep }}}$ de $K_{\text {sep }}$ et que si $L / K$ est finie, $\widetilde{N}_{L / K}$ désigne la norme $N_{\widetilde{L} / \widetilde{K}}$.

Un étiquetage de Lubin-Tate sur $K$ a été défini par Koch et de Shalit dans [KdS96] comme la donnée d'une uniformisante $\pi_{L}$ de $\widetilde{L}$ pour toute extension séparable finie $L$ de $K$ de sorte que, pour toute extension séparable finie $M \supset L, \pi_{L}=\widetilde{N}_{M / L}\left(\pi_{M}\right)$. Mais dans cet article, on n'utilisera que l'étiquetage de Lubin-Tate qui coïncide avec la famille projective $\left\{\varpi_{E} \in E ; E \in \mathcal{F}_{K_{\text {sep }}^{\langle\phi\rangle} / K}\right\}$ du lemme 1 , sur $\mathcal{F}_{K_{\text {sep }} / K}$; son existence et son unicité sont garanties par le fait que, pour toute extension séparable finie $L$ de $K$, il existe $E \in \mathcal{F}_{K_{s e p}^{\langle\phi\rangle} / K}$ telle que $L \subset E_{n r}$.

\section{DÉFINITION 1.}

(1) Soit $L$ une extension séparable finie de $K$. On appelle étiquette de $L$ et on note $\varpi_{L}$ l'uniformisante de $L_{n r}$ provenant de l'étiquetage ci-dessus. (Remarquons que si $M / L$ est finie non ramifiée alors $\left.\varpi_{M}=\varpi_{L}\right)$.

(2) Soit $L$ une extension séparable infinie de $K$; on définit l'étiquette $\varpi_{L}$ de $L$ comme suit : si l'indice de ramification de $L / K$ est fini, il existe une extension finie $L_{0} / K$ telle que $L$ soit une extension non ramifiée de $L_{0}$ et $\varpi_{L}=\varpi_{L_{0}}$; dans le cas général, on définit l'étiquette $\varpi_{L}$ comme la famille projective $\left\{\varpi_{E_{n r}}\right\}_{E \in \mathcal{F}_{L / K}}$.

\section{Remarques.}

(1) Si $M / L$ est non ramifiée alors $\varpi_{M}=\varpi_{L}$.

(2) Si $L$ est une extension arithmétiquement profinie de $K$ alors $\varpi_{L}$ est une uniformisante du corps des normes $\mathfrak{N}_{\widetilde{K}}(\widetilde{K} L)$ et si $M$ est une extension arithmétiquement profinie de $K$ contenant $L$ alors $\widetilde{N}_{M / L}\left(\varpi_{M}\right)=\varpi_{L}$; pour ce qui concerne la projection canonique $\widetilde{N}_{M / L}$, voir $\S 1.3$. 


\section{UNE THÉORIE DU CORPS DE CLASSES LOCAL NON ABÉLIEN}

\subsubsection{Extensions compatibles.}

DÉfinition 2. Une extension séparable $L / K$ est dite compatible si elle est de degré résiduel $f$ fini et si elle est fixe par $\phi^{f}$.

\section{Remarques.}

(1) Les extensions galoisiennes compatibles de $K$ sont les extensions galoisiennes de la forme $K^{\prime} L / K$ où $K^{\prime} / K$ est finie non ramifiée et où $L \subset K_{\text {sep }}^{\langle\phi\rangle}$.

(2) Si $L / K$ est une extension compatible finie de degré résiduel $d$ alors les extensions de $L$ compatibles relativement au dévissage de Lubin-Tate $\phi_{L}=\phi^{d}$ sur $L$ sont celles qui sont compatibles relativement à $\phi$ sur $K$.

(3) Toute extension galoisienne finie $L$ de $K$ est contenue dans $L_{n r}^{\prime}$ où $L^{\prime}=L_{n r} \cap K_{\text {sep }}^{\langle\phi\rangle}$ et il existe une extension finie non ramifiée de $L$, compatible (et galoisienne sur $K$ ). Voir [KdS96, Section 0.4].

(4) Soit $L / K$ une extension arithmétiquement profinie et compatible ; l'équivalence de catégories de Wintenberger $\mathfrak{N}_{L / K}$ (voir $\S 1.2$ ) fournit un dévissage de Lubin-Tate $\mathfrak{N}_{L / K}\left(\phi_{L}\right)$ dont le corps des invariants est $\mathfrak{N}_{L / K}\left(K_{\text {sep }}^{\left\langle\phi_{L}\right\rangle}\right)$ et pour lequel les extensions compatibles sur le corps de normes $\mathfrak{N}_{K}(L)$ sont les $\mathfrak{N}_{L / K}(E), E$ parcourant les extensions de $K$ contenant $L$ et compatibles pour $\phi$.

De plus si $\mathfrak{E}=\mathfrak{N}_{L / K}(E)$ est une extension séparable du corps des normes $\mathfrak{N}_{K}(L)$ l'étiquette de $\mathfrak{E}$ relativement au dévissage de Lubin-Tate $\mathfrak{N}_{L / K}\left(\phi_{L}\right)$ sur $\mathfrak{N}_{K}(L)$ est $\varpi_{\mathfrak{E}}=\varpi_{E}$.

Dorénavant on s'autorisera l'abus de langage $\mathfrak{N}_{L / K}(\phi)=\phi$; c'est un automorphisme de $\mathfrak{N}_{K}(L)_{\text {sep }}=\mathfrak{N}_{L / K}\left(K_{\text {sep }}\right)$ qui se prolonge par continuité à son complété.

Lemme 2. Soit $L$ une extension arithmétiquement profinie de $K$. Si $L / K$ est compatible alors $\varpi_{L} \in \mathfrak{N}_{K}(L)$; si, de plus, $L / K$ est galoisienne alors $\phi\left(\varpi_{L}\right)=\varpi_{L}$.

Démonstration. Il suffit de démontrer le lemme pour des extensions $L / K$ finies.

Si l'extension $L / K$ est galoisienne, par restriction à $L, \phi$ engendre un groupe fini de $K$-automorphismes de $L$ dont le corps des invariants est $L \cap K_{\text {sep }}^{\langle\phi\rangle}$ et si $L / K$ est compatible alors l'extension $L / L \cap K_{\text {sep }}^{\langle\phi\rangle}$ est non ramifiée et donc $\varpi_{L}=\varpi_{L \cap K_{\text {sep }}\langle\phi\rangle} \in L \cap K_{\text {sep }}^{\langle\phi\rangle}$.

Plus généralement, soit $L / K$ une extension compatible finie et soit $M$ une extension galoisienne, finie et compatible sur $K$ contenant $L$ (Remarque 3 ci-dessus). Soit $d_{L}$ (respectivement $d_{M}$ ) le degré résiduel de $L$ (respectivement $M$ ) sur $K$, l'extension $L K_{d_{M}} / L$ est non ramifiée donc $\varpi_{L}=\varpi_{L K_{d_{M}}}=$ $N_{M / L K_{d_{M}}}\left(\varpi_{M}\right) \in L K_{d_{M}}$ mais $\varpi_{M} \in M \cap K_{\text {sep }}^{\langle\phi\rangle}$ et les extensions $M \cap K_{\text {sep }}^{\langle\phi\rangle}$ et $L K_{d_{M}}$ sont linéairement disjointes sur $L K_{d_{M}} \cap K_{\text {sep }}^{\langle\phi\rangle}$ donc $\varpi_{L}=N_{M \cap K_{\text {sep }}^{\langle\phi\rangle} / L K_{d_{M}} \cap K_{\text {sep }}^{\langle\phi\rangle}}\left(\varpi_{M}\right) \in L K_{d_{M}} \cap K_{\text {sep }}^{\langle\phi\rangle} \subset L$.

2.1.3 Groupes étiquetés. Soit $L$ une extension finie compatible de $K$ à corps résiduel $\mathbb{F}_{q_{L}}$ et soit $\pi$ une uniformisante de $L$. On note $\left(U^{i}(L)\right)_{i \geqslant 1}$ la filtration naturelle du groupe des unités $U(L)$ et $L_{i)}$ le corps de classes du sous-groupe $\langle\pi\rangle U^{i}(L)$ du complété profini $\widehat{L^{\times}}$du groupe multiplicatif de $L$; alors $L_{i}$ est une extension finie totalement ramifiée de $L$ de degré $\left(q_{L}-1\right) q_{L}^{i-1}$. L'extension abélienne maximale $L_{a b}$ de $L$ n'est autre que $L_{n r} L_{\pi}$ où $L_{\pi}=\bigcup_{i \geqslant 1} L_{i \text { ) }}$.

Si $F$ est une loi de groupe formel de Lubin-Tate sur $L$ attachée à $\pi$, pour $a \in \mathcal{O}(L)$, $[a]_{F}$ désigne l'endomorphisme de $F$ tel que $[a]_{F}(X) \equiv a X \bmod X^{2}$ et qui commute avec $[\pi]_{F}$; on a $L_{i}=L\left(\omega_{i}\right)$ où $\omega_{i}$ est un point de torsion de niveau $i$ de $F$ c'est à dire tel que $\left[\pi^{i}\right]_{F}\left(\omega_{i}\right)=0$ et $\left[\pi^{i-1}\right]_{F}\left(\omega_{i}\right) \neq 0$. Une suite d'éléments de torsion $\omega_{i}$ de niveau $i$ telle que $[\pi]_{F}\left(\omega_{i}\right)=\omega_{i-1}$ pour tout $i \geqslant 2$ et $[\pi]_{F}\left(\omega_{1}\right)=0$ est un générateur du module de Tate de $F$. 


\section{F. LAUBiE}

Lemme 3 (Koch et de Shalit [KdS96, Section 0.3]). Il existe une unique loi de groupe formel de Lubin-Tate $F_{L}$ définie sur l'anneau des entiers de $L$ attachée à l'étiquette $\varpi_{L}$ dont un générateur du module de Tate soit l'étiquette de l'extension abélienne maximale totalement ramifiée $L_{\varpi}$, c'est à dire telle que $\left[\varpi_{L}\right]_{F_{L}}\left(\varpi_{\left.L_{i}\right)}\right)=\varpi_{\left.L_{i-1}\right)}$ pour tout $i \geqslant 2$ et $\left[\varpi_{L}\right]_{F_{L}}\left(\varpi_{L_{1)}}\right)=0$.

Remarque et définition 3. Dans le cas plus général où $L$ est le corps des normes d'une extension arithmétiquement profinie compatible de $K$, le dévissage de Lubin-Tate sur $L$ et les étiquettes des extensions séparables de $L$ sont uniquement déterminés par $\phi$ et donc il existe également un unique groupe formel de Lubin-Tate associé à $\varpi_{L}$ et tel que $\left[\varpi_{L}\right]_{F_{L}}\left(\varpi_{\left.L_{i}\right)}\right)=\varpi_{L_{i-1}}$ pour tout $i \geqslant 2$ et $\left[\varpi_{L}\right]_{F_{L}}\left(\varpi_{\left.L_{1}\right)}\right)=0$.

On dit que $F_{L}$ est le groupe formel étiqueté (ou simplement le groupe étiqueté) sur $L$.

\subsection{Groupes et applications de réciprocité de Koch-de Shalit}

2.2.1 Définitions. Cette section développe les $\S \S 1.5$ et 1.6 ci-dessus. On en reprend intégralement les hypothèses et notations. Rappelons seulement que la notation $G=\operatorname{Aut}_{\mathfrak{g}_{L}}\left(L_{a b} / L\right)$ a déjà été donnée au $\S 1.2$ et que $G=\operatorname{Gal}\left(\mathcal{L}_{a b} / K\right)=\operatorname{Gal}\left(L_{a b} / K\right)$ dans le cas où l'extension $\mathcal{L} / K$ est finie.

La définition (voir $\S 1.5$ ) du groupe de Koch-de Shalit a été originellement donnée (voir [KdS96, Section 2.2]) dans le cas où $\mathcal{L}$ (ou $L$ ) est une extension finie de $K$. Remplacer $L$ par le corps des normes d'une extension arithmétiquement profinie et infinie de $K$, revient à associer à $L$, non plus un groupe fini, mais un groupe infini compact $\mathfrak{g}_{L}$ d'automorphismes de $L$. Cela ne change formellement rien à la définition du groupe de Koch-de Shalit $G\left(\mathfrak{g}_{L}, \varpi_{L}\right)$.

De même, le théorème 1 a été établi dans le cas où le groupe d'automorphismes $\mathfrak{g}_{L}$ est fini [KdS96, Proposition 2.4]. Il reste valide dans le cas général avec la même démonstration.

Démonstration de la proposition 1. Le diagramme de la proposition 1 (voir $\S 1.6$ ) est l'image par le foncteur $\mathfrak{N}_{\mathcal{L} / K}$ du diagramme suivant.

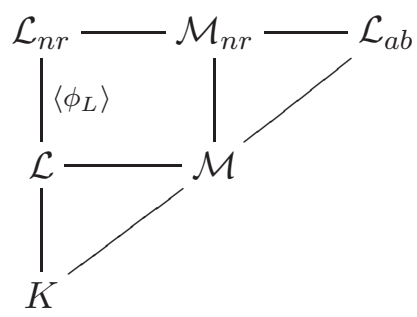

Le groupe de Galois de $\mathcal{L}_{a b} / \mathcal{M}$ est le sous-groupe fermé normal de $\operatorname{Gal}\left(\mathcal{L}_{a b} / K\right)$ engendré par $\phi_{L}$; il contient le sous-groupe fermé engendré par les commutateurs de $\phi_{L}:\left[\operatorname{Gal}\left(\mathcal{L}_{a b} / K\right),\left\langle\phi_{L}\right\rangle\right]$ qui est normal dans $\operatorname{Gal}\left(\mathcal{L}_{a b} / K\right)$; or, d'une part, $\operatorname{Gal}\left(\mathcal{L}_{a b} / \mathcal{M}\right) \subset\left\langle\phi_{L}\right\rangle \times\left[\operatorname{Gal}\left(\mathcal{L}_{a b} / K\right),\left\langle\phi_{L}\right\rangle\right]$ et, d'autre part, $\operatorname{Gal}\left(\mathcal{M}_{n r} / K\right)$ est une extension centrale de $\operatorname{Gal}(\mathcal{M} / K)$ par $\left\langle\phi_{L}\right\rangle$; il s'ensuit que $\left[\operatorname{Gal}\left(\mathcal{L}_{a b} / K\right),\left\langle\phi_{L}\right\rangle\right]=\operatorname{Gal}\left(\mathcal{L}_{a b} / \mathcal{M}_{n r}\right)$ et

$$
\operatorname{Gal}\left(\mathcal{L}_{a b} / \mathcal{M}\right)=\left\langle\phi_{L}\right\rangle \times\left[\operatorname{Gal}\left(\mathcal{L}_{a b} / K\right),\left\langle\phi_{L}\right\rangle\right]
$$

autrement dit

$$
\operatorname{Gal}\left(L_{a b} / M\right)=\left\langle\phi_{L}\right\rangle \times\left[G,\left\langle\phi_{L}\right\rangle\right]
$$

Par la théorie du corps de classes local (abélien), l'image de $\left[G,\left\langle\phi_{L}\right\rangle\right]$ par l'application de réciprocité d'Artin sur $L$ est le sous-groupe fermé $V_{\mathfrak{g}_{L}}\left(\varpi_{L}\right)$ de $U(L)$ engendré par $\left\{g\left(\varpi_{L}\right) / \varpi_{L} ; g \in \mathfrak{g}_{L}\right\}$, donc, par le théorème 1 , l'image de $\operatorname{Gal}\left(L_{a b} / M\right)$ par $\iota_{L}$ est $\left\{\phi_{L}^{\nu}\right\}_{\nu \in \widehat{\mathbb{Z}}} \times V_{\mathfrak{g}_{L}}\left(\varpi_{L}\right)$.

Corollaire. Les assertions suivantes sont équivalentes:

(i) l'extension $\mathcal{L}_{a b}^{\left\langle\phi_{L}\right\rangle} / K$ est galoisienne ;

(ii) $\bar{G}=G /\left\langle\phi_{L}\right\rangle$;

(iii) l'extension $\mathcal{L} / K$ est non ramifiée. 


\section{UNE THÉORIE DU CORPS DE CLASSES LOCAL NON ABÉLIEN}

Démonstration. Les assertions (i) et (ii) sont équivalentes à $V_{\mathfrak{g}_{L}}\left(\varpi_{L}\right)=(1)$ ce qui se produit uniquement quand $\varpi_{L}$ est fixe par $\mathfrak{g}_{L}$ c'est à dire quand $\varpi_{L}=\varpi_{K}$ et cette dernière assertion signifie que $\mathcal{L} / K$ est non ramifiée.

2.2.2 Normes de Koch-de Shalit. Cette section est le développement du $\S 1.8$ ci-dessus. On en reprend intégralement les hypothèses et notations. Son objet est la démonstration d'une partie de la proposition 3 qui servira par la suite à démontrer la proposition 2.

La définition de l'analogue de la norme $r_{L^{\prime} / L}$ dans les groupes de Koch-de Shalit a été donnée (voir [KdS96, Section 2.2]) dans le cas où $L^{\prime}=\mathcal{L}^{\prime}$ est une extension galoisienne, compatible et finie de $K$ qui contient $L=\mathcal{L}$ également galoisienne et compatible sur $K$. Dans ce cas

$$
\begin{gathered}
r_{L^{\prime} / L}: G\left(\mathfrak{g}_{L^{\prime}}, \varpi_{L^{\prime}}\right) \rightarrow G\left(\mathfrak{g}_{L}, \varpi_{L}\right), \\
\left(\gamma^{\prime}, b^{\prime}\right) \mapsto(\gamma, b)
\end{gathered}
$$

où $\gamma=\left.\gamma^{\prime}\right|_{L_{n r}}$ et où $b=\prod_{0 \leqslant i<d} \phi_{L}^{i}\left(\tilde{N}_{L^{\prime} / L}\left(b^{\prime}\right)\right)$.

On rappelle que $\widetilde{N}_{L^{\prime} / L}$ est l'application norme dans l'extension finie $\widetilde{L}^{\prime} / \widetilde{L}$.

Koch et de Shalit ont montré que $r_{L^{\prime} / L}$ est un homomorphisme de groupes surjectif dont la restriction au sous-groupe $U\left(L^{\prime}\right)$ de $G\left(\mathfrak{g}_{L^{\prime}}, \varpi_{L^{\prime}}\right)$ coïncide avec la restriction à $U\left(L^{\prime}\right)$ de la norme de l'extension $L^{\prime} / L$ et que le diagramme suivant commute.

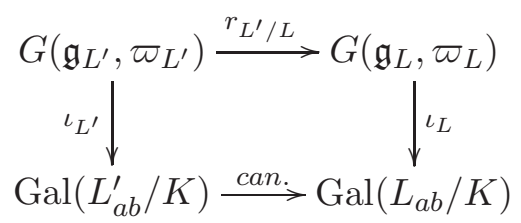

Dans le cas où $\mathcal{L}$ et $\mathcal{L}^{\prime}$ sont des extensions galoisiennes, compatibles, arithmétiquement profinies et infinies de $K$ telles que $\mathcal{L} \subset \mathcal{L}^{\prime}, L$ et $L^{\prime}$ désignent leur corps de normes respectif. Supposons, en outre, que l'extension $\mathcal{L} / \mathcal{L}^{\prime}$ soit finie, alors $L^{\prime}$ est une extension finie de $L$ et la définition de $r_{L^{\prime} / L}: G\left(\mathfrak{g}_{L^{\prime}}, \varpi_{L^{\prime}}\right) \rightarrow G\left(\mathfrak{g}_{L}, \varpi_{L}\right)$ est exactement la même que dans le cas précédent. De plus, le diagramme suivant commute.

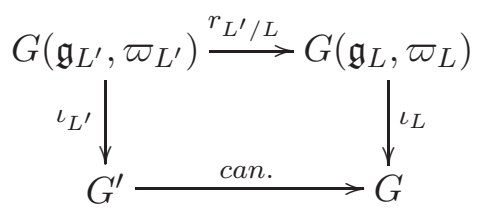

Comme pour le théorème 1, la démonstration de Koch et de Shalit [KdS96, Sections 2.11 et 2.12] fonctionne sans aucune modification avec ces hypothèses. De plus, il ne semble pas que l'appartenance des étiquettes aux corps de base (qui n'est pas supposée par Koch et de Shalit) puisse simplifier cette démonstration.

Lorsque l'extension finie $\mathcal{L}^{\prime} / \mathcal{L}$ est abélienne, le résultat est reformulé comme suit.

Lemme 4. Supposons que $L^{\prime} / L$ soit une extension abélienne finie. Soit $\gamma^{\prime} \in \Gamma^{\prime}$. Il existe $b^{\prime} \in U\left(\widetilde{L}^{\prime}\right)$ tel que $\phi_{L^{\prime}}\left(b^{\prime}\right) / b^{\prime}=\gamma^{\prime}\left(\varpi_{L^{\prime}}\right) / \varpi_{L^{\prime}}$ et on a $\gamma^{\prime}=\iota_{L}\left(\left.\gamma^{\prime}\right|_{L_{n r}}, b\right)$ avec $b=\prod_{0 \leqslant i<d} \phi_{L^{i}}^{i} \tilde{N}_{L^{\prime} / L}\left(b^{\prime}\right)$.

Remarque (Koch et de Shalit [KdS96, Remark 2.12]). Si, en plus des hypothèses du lemme $4, L^{\prime} / L$ est totalement ramifiée et $\left.\mathrm{si} \gamma^{\prime}\right|_{L_{n r}}=\mathrm{id}_{L_{n r}}$, alors on retrouve un théorème de Dwork : $\left.\gamma^{\prime}\right|_{L^{\prime}}=$ $\left.\iota_{L}(1, b)\right|_{L^{\prime}}=\left(b^{-1}, L^{\prime} / L\right)$ avec $b=\widetilde{N}_{L^{\prime} / L}\left(b^{\prime}\right)$.

Avant de généraliser ce qui précède au cas où l'extension galoisienne $\mathcal{L}^{\prime} / \mathcal{L}$ est infinie, il faut préciser le comportement de l'application de réciprocité de Koch-de Shalit avec le foncteur de Wintenberger. 


\section{F. LAUBiE}

2.2.3 Corps de normes et corps de classes commutent. Comme précédemment, $\mathcal{L} / K$ est une extension galoisienne, compatible, arithmétiquement profinie et infinie, $d_{L}$ est son degré résiduel, $\mathcal{F}_{\mathcal{L} / K}$ est la famille des sous-extensions finies $E / K$ de $\mathcal{L} / K$ et $L$ est le corps des normes $\mathfrak{N}_{K}(\mathcal{L})$.

Considérons les images par le foncteur de Wintenberger $\mathfrak{N}_{\mathcal{L} / K}$ de certains corps de classes. On a $\mathfrak{N}_{\mathcal{L} / K}\left(\bigcup_{E \in \mathcal{F}_{\mathcal{L} / K_{d_{L}}}} E_{a b}^{\left\langle\phi_{E}\right\rangle}\right)=L_{a b}^{\left\langle\phi_{L}\right\rangle}, L_{a b}^{\left\langle\phi_{L}\right\rangle}$ (respectivement $E_{a b}^{\left\langle\phi_{E}\right\rangle}$ ) étant le corps de classes du sous-groupe $\left\langle\varpi_{L}\right\rangle$ de $\widehat{L^{\times}}$(respectivement du sous-groupe $\left\langle\varpi_{E}\right\rangle$ de $\widehat{E^{\times}}$).

En fait, on peut montrer un résultat légèrement plus précis : soit $i \geqslant 1$ et soit $L_{i}$ ) (respectivement $E_{i}$ ) le corps de classes du sous-groupe $\left\langle\varpi_{L}\right\rangle U^{i}(L)$ (respectivement $\left\langle\varpi_{E}\right\rangle U^{i}(E)$ ) de $\widehat{L^{x}}$ (respectivement de $\widehat{E^{\times}}$). Pour tous $E, E^{\prime} \in \mathcal{F}_{\mathcal{L} / K_{d_{L}}}$ dont le plus petit nombre de ramification est $\geqslant i$ et tels que $E \subset E^{\prime}$, on a $E_{i)} \subset E_{i)}^{\prime}$; donc pour $E$ parcourant l'ensemble de ces corps, on a $L_{i)}=\mathfrak{N}_{\mathcal{L} / K}\left(\bigcup_{E} E_{i)}\right)$.

Considérons le système projectif des groupes $G\left(\mathfrak{g}_{E}, \varpi_{E}\right)$, où $\mathfrak{g}_{E}=\operatorname{Gal}(E / K), E$ parcourant les sous-extensions galoisiennes finies de $\mathcal{L} / K$ contenant $K_{d_{L}}$, pour les morphismes de transition $r_{E^{\prime} / E}$. Une telle extension $E$ est compatible sur $K$. D'autre part, $\mathfrak{N}_{\mathcal{L} / K}\left(\lim _{\longleftarrow} \Gamma_{E}\right)=\mathfrak{N}_{\mathcal{L} / K}\left(\operatorname{Gal}\left(\mathcal{L}_{n r} / K\right)\right)=$ $\Gamma_{L}$ et $\lim _{\longleftarrow} G\left(\mathfrak{g}_{E}, \varpi_{E}\right)$ s'identifie canoniquement à $G\left(\mathfrak{g}_{L}, \varpi_{L}\right)$. On a donc défini deux applications :

$$
\begin{gathered}
\iota_{L}: G\left(\mathfrak{g}_{L}, \varpi_{L}\right) \rightarrow G=\operatorname{Aut}_{\mathfrak{g}_{L}}\left(L_{a b} / L\right) \\
\lim _{\longleftarrow} \iota_{E}: G\left(\mathfrak{g}_{L}, \varpi_{L}\right) \rightarrow \underset{\longleftarrow}{\lim } \operatorname{Gal}\left(E_{a b} / K\right)=\operatorname{Gal}\left(\mathcal{L}_{a b} / K\right) .
\end{gathered}
$$

Proposition 4. Nous avons $\iota_{L}=\mathfrak{N}_{\mathcal{L} / K}\left(\varliminf_{\longleftarrow} \iota_{E}\right)$.

Démonstration. Soit $\iota=\mathfrak{N}_{\mathcal{L} / K}\left(\lim _{\longleftarrow} \iota_{E}\right)$ et soit $(\gamma, b) \in G(\mathfrak{g}, \pi)$. Il suffit de montrer que les restrictions de $\iota(\gamma, b)$ et de $\iota_{L}(\gamma, b)$ à toute extensions abéliennes finies de $L_{n r}$ coïncident. Mais une telle extension est toujours contenue dans un corps de normes $\widetilde{\mathfrak{N}}_{K}\left(\mathcal{L}^{\prime}\right)$ où $\mathcal{L}^{\prime}$ est une extension abélienne finie de $\mathcal{L}$ galoisienne sur $K$ et compatible. Soit donc $L^{\prime}=\mathfrak{N}_{K}\left(\mathcal{L}^{\prime}\right) / L$ abélienne, finie, compatible et montrons que $\left.\iota(\gamma, b)\right|_{L_{n r}^{\prime}}=\left.\iota_{L}(\gamma, b)\right|_{L_{n r}^{\prime}}$.

L'ensemble $\mathcal{F}^{\prime}$ des sous-extensions galoisiennes finies $F / K$ de $\mathcal{L} / K$, de degré résiduel $d_{L}$, pour lesquelles il existe une extension abélienne finie $E_{F}$ de $F$ galoisienne sur $K$, linéairement disjointe de $\mathcal{L} / F$ et telle que $\mathcal{L}^{\prime}=E_{F} \mathcal{L}$, est cofinal dans $\mathcal{F}_{\mathcal{L} / K} ;$ l'ensemble $\left\{E_{F} ; F \in \mathcal{F}^{\prime}\right\}$ est cofinal dans $\mathcal{F}_{\mathcal{L}^{\prime} / K}$. Alors $\gamma^{\prime}=\mathfrak{N}_{\mathcal{L} / K}^{-1}\left(\iota_{L}(\gamma, b)\right)$ est un automorphisme de $\mathcal{L}_{a b}$ et on note $\gamma_{E_{F}}^{\prime}$ (respectivement $\gamma_{F}$ ) sa restriction à $\left(E_{F}\right)_{n r}$ (respectivement à $\left.F_{n r} \in \mathcal{F}^{\prime}\right)$. Il suffit de montrer que $\gamma_{E_{F}}^{\prime}$ est la restriction à $\left(E_{F}\right)_{n r}$ de $\iota_{F}\left(\gamma_{F}, \widetilde{N}_{L / F}(b)\right)$.

Soit $d$ le degré résiduel de $L^{\prime} / L$. Par le lemme 4 , il existe $b^{\prime} \in U\left(\widetilde{L}^{\prime}\right)$ tel que $\left(\mathfrak{N}_{\mathcal{L} / K}\left(\gamma^{\prime}\right), b^{\prime}\right) \in$ $G\left(\mathfrak{g}_{L^{\prime}}, \varpi_{L^{\prime}}\right)$ et $b=\prod_{0 \leqslant i<d} \phi^{i}\left(\tilde{N}_{L^{\prime} / L}\left(b^{\prime}\right)\right)$. Soient $b_{E_{F}}^{\prime}=\widetilde{N}_{L^{\prime} / E_{F}}\left(b^{\prime}\right)$ et $b_{F}=\prod_{0 \leqslant i<d} \phi^{i}\left(\widetilde{N}_{E_{F} / F}\left(b_{E_{F}}^{\prime}\right)\right)$. On a alors $b_{F}=\widetilde{N}_{L / F}(b)$ et $\left.\iota_{F}\left(\gamma_{F}, b_{F}\right)\right|_{E_{F}}=\gamma_{E_{F}}^{\prime}$.

Remarque. Cette proposition généralise le théorème 3.2.2 de [Lau88].

2.2.4 Norme de Koch-de Shalit (suite). Les notations et hypothèses de cette section sont toujours celles du $\S 1.8$ et du $\S 2.2 .2$.

Rappelons que dans le cas où $\mathcal{L}$ et $\mathcal{L}^{\prime}$ sont des extensions de $K$, galoisiennes, arithmétiquement profinies, compatibles telles que $\mathcal{L}^{\prime} / \mathcal{L}$ soit une extension infinie, la norme de Koch-de Shalit est :

$$
\begin{gathered}
r_{L^{\prime} / L}: G\left(\mathfrak{g}_{L^{\prime}}, \varpi_{L^{\prime}}\right) \rightarrow G\left(\mathfrak{g}_{L}, \varpi_{L}\right), \\
\left(\gamma^{\prime}, b^{\prime}\right) \mapsto(\gamma, b)
\end{gathered}
$$

avec, pour $\gamma^{\prime}=\mathfrak{N}_{\mathcal{L}^{\prime} / K}(g) \in \Gamma^{\prime}, \gamma=\mathfrak{N}_{\mathcal{L} / K}\left(\left.g\right|_{\mathcal{L}_{n r}}\right), g \in \operatorname{Gal}\left(\mathcal{L}_{n r}^{\prime} / K\right)$ et $b=\prod_{0 \leqslant i<d} \phi_{L}^{i}\left(\widetilde{N}_{L^{\prime} / L}\left(b^{\prime}\right)\right)$, $\widetilde{N}_{L^{\prime} / L}=\tilde{N}_{\mathcal{L}^{\prime} / \mathcal{L}}$ étant la projection canonique (voir $\S 1.3$ ). 
Proposition 5. Le diagramme suivant commute.

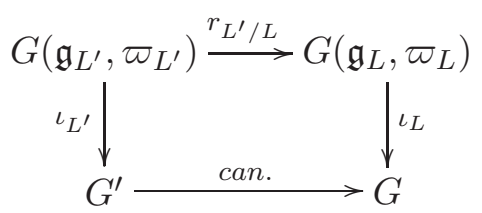

Démonstration. Dans le cas où $\mathcal{L}$ est une extension finie de $K$ telle que $\mathcal{L}^{\prime} / \mathcal{L}$ est totalement ramifiée, c'est exactement la proposition 4 . Dans le cas où $\mathcal{L}$ est une extension finie compatible de $K$ contenue dans $\mathcal{L}^{\prime}$, on se ramène au cas précédent en considérant une sous-extension finie $\mathcal{L}^{\prime \prime}$ de $\mathcal{L}^{\prime} / K$ contenant $\mathcal{L}$ et telle que $\mathcal{L}^{\prime} / \mathcal{L}^{\prime \prime}$ soit totalement ramifiée $: r_{L^{\prime} / L}=r_{L^{\prime} / L^{\prime \prime}} \circ r_{L^{\prime \prime} / L}$ et la proposition 5 est vraie pour $r_{L^{\prime} / L^{\prime \prime}}$ et pour $r_{L^{\prime \prime} / L}$. Enfin dans le cas où $\mathcal{L}^{\prime} / \mathcal{L}$ est une extension infinie, il suffit de passer à la limite projective les diagrammes correspondant à $r_{E / \mathcal{L}}$ pour $E \in \mathcal{F}_{\mathcal{L}^{\prime} / \mathcal{L}}$ et d'appliquer à nouveau la proposition 4.

Proposition 6. Le diagramme suivant commute.

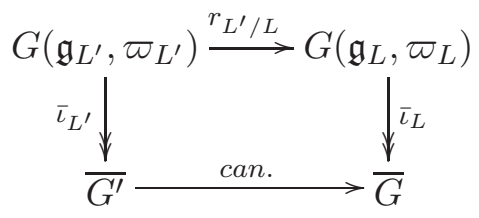

Démonstration. Bien qu'elle ne soit pas vraiment nécessaire, les quelques précisions qui suivent sont utiles. Par la proposition 1 , le noyau de $\bar{\iota}_{L^{\prime}}\left(\right.$ respectivement de $\left.\bar{\iota}_{L}\right)$ est $\phi_{L^{\prime}}^{\widehat{\mathbb{Z}}} \times V_{\mathfrak{g}_{L^{\prime}}}\left(\varpi_{L^{\prime}}\right)$ (respectivement $\left.\phi_{L}^{\widehat{\mathbb{Z}}} \times V_{\mathfrak{g}_{L}}\left(\varpi_{L}\right)\right)$ avec $V_{\mathfrak{g}_{L^{\prime}}}\left(\varpi_{L^{\prime}}\right) \subset U\left(L^{\prime}\right)$ (respectivement $\left.V_{\mathfrak{g}_{L}}\left(\varpi_{L}\right) \subset U(L)\right)$. La projection canonique $\widetilde{N}_{L^{\prime} / L}$ applique $U\left(L^{\prime}\right)$ dans $U(L)$ et $V_{\mathfrak{g}_{L^{\prime}}}\left(\varpi_{L^{\prime}}\right)$ dans $V_{\mathfrak{g}_{L}}\left(\varpi_{L}\right)$. Donc $r_{L^{\prime} / L}$ applique $V_{\mathfrak{g}_{L^{\prime}}}\left(\varpi_{L^{\prime}}\right)$ dans $V_{\mathfrak{g}_{L}}\left(\varpi_{L}\right)^{d} \subset V_{\mathfrak{g}_{L}}\left(\varpi_{L}\right)$ où $d$ est le degré résiduel de $\mathcal{L}^{\prime} / \mathcal{L}$.

2.2.5 Changement $d u$ corps de base. Soit $K^{\prime} / K$ une extension compatible, finie, de degré résiduel $f$. On note $\left(K^{\prime}, \phi^{\prime}\right)$ le dévissage de Lubin-Tate canoniquement déduit de $(K, \phi)$, c'est à dire avec $\phi^{\prime}=\phi^{f}$.

On suppose que $\mathcal{L}$ est une extension galoisienne, compatible, arithmétiquement profinie de $K$ qui contient $K^{\prime}$ de sorte qu'elle est également compatible sur $K^{\prime}$ relativement à $\phi^{\prime}$ avec $\phi_{L}^{\prime}=\phi_{L}$; en outre les corps de normes $L=\mathfrak{N}_{K}(L)$ et $\mathfrak{N}_{K^{\prime}}(L)$ s'identifient. On note $\mathfrak{g}_{L}^{\prime}=\mathfrak{N}_{K^{\prime}}\left(\mathrm{Gal}\left(\mathcal{L} / K^{\prime}\right), \Gamma^{\prime}=\right.$ $\operatorname{Aut}_{\mathfrak{g}_{L}^{\prime}}\left(L_{n r} / L\right), G^{\prime}=\operatorname{Aut}_{\mathfrak{g}_{L}^{\prime}}\left(L_{a b} / L\right), M^{\prime}=\mathfrak{N}_{L / K^{\prime}}\left(\mathcal{M}^{\prime}\right)$ où $\mathcal{M}^{\prime} / K^{\prime}$ est la sous-extension galoisienne maximale de $\mathcal{L}_{a b} / K^{\prime}$ fixe par $\phi_{L}$ et $\bar{G}^{\prime}=\operatorname{Aut}_{\mathfrak{g}_{L}^{\prime}}\left(M^{\prime} / L\right)$. (Plus généralement, si $\mathfrak{O}$ est un objet relatif à $K, \mathfrak{O}^{\prime}$ désigne l'objet correspondant relatif à $K^{\prime}$ ).

Proposition 7. Le diagramme suivant commute.

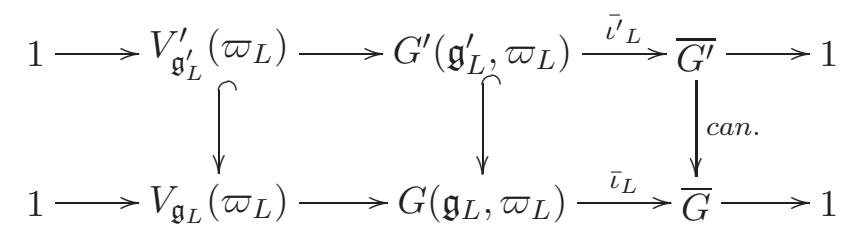

2.2.6 Calculs de l'application de réciprocité de Koch-de Shalit. On rappelle que $L$ désigne toujours soit une extension galoisienne, finie et compatible de $K$, soit le corps des normes d'une extension $\mathcal{L}$ galoisienne, infinie, arithmétiquement profinie et compatible de $K$. Dans les deux cas, soit $\mathbb{F}_{q_{L}}$ avec $q_{L}=q^{d_{L}}$, le corps résiduel de $L$. On rappelle que $F_{L}$ désigne le groupe étiqueté sur $L$, que $\overline{F_{L}}$ est sa réduction modulo $\varpi_{L}$ (coefficient par coefficient) et que, pour $\gamma \in \Gamma, \gamma F_{L}$ est l'image de $F_{L}$ coefficient par coefficient, par $\gamma$. 


\section{F. LAUbie}

Soit $(\gamma, b) \in G\left(\mathfrak{g}_{L}, \varpi_{L}\right)$. On sait que $\iota_{L}(\gamma, b) \in \operatorname{Aut}_{\mathfrak{g}_{L}}\left(L_{a b} / L\right)$ est l'unique automorphisme de $L_{a b}$ qui prolonge $\gamma \in \operatorname{Aut}_{\mathfrak{g}_{L}}\left(L_{n r} / L\right)$ et qui applique $\varpi_{\left.L_{i}\right)} \operatorname{sur} \phi_{L}^{1-i}[b]_{F_{L}, \gamma F_{L}}\left(\varpi_{\left.L_{i}\right)}\right)$, pour tout $i \geqslant 1$, où $L_{i)}$ désigne le corps de classes du sous-groupe $\left\langle\varpi_{L}\right\rangle U^{i}(L)$ de $\widehat{L^{\times}}$et où $[b]_{F_{L}, \gamma F_{L}}(X) \in \mathcal{O}(\widetilde{L})[[X]]$ est l'unique isomorphisme de groupes formels de $F_{L} \operatorname{sur} \gamma F_{L}$ défini $\operatorname{sur} \mathcal{O}(\widetilde{L})$ tel que $[b]_{F_{L}, \gamma F_{L}}(X) \equiv b X$ $\bmod X^{2}$ et que $[b]_{F_{L}, \gamma F_{L}} \circ\left[\varpi_{L}\right]_{F_{L}}=\left[\varpi_{L}\right]_{\gamma F_{L}} \circ[b]_{F_{L}, \gamma F_{L}}$.

On se propose de calculer $\mathfrak{N}_{\widetilde{L} L_{a b} / \widetilde{L}}\left(\iota_{L}(\gamma, b)\right)\left(\varpi_{L_{a b}}\right)$ puis $\mathfrak{N}_{M / L}\left(\bar{\iota}_{L}(\gamma, b)\right)\left(\varpi_{M}\right)$, c'est à dire de démontrer la proposition 2 .

Démonstration de la proposition 2. Montrons que $\mathfrak{N}_{\widetilde{L} L_{a b} / \widetilde{L}}\left(\iota_{L}(\gamma, b)\right)\left(\varpi_{L_{a b}}\right)=\{b\}_{\nu_{\gamma}}\left(\varpi_{L_{a b}}\right)$ où $\{b\}_{\nu_{\gamma}}$ est la réduction $\overline{[b]_{F_{L}, \gamma F_{L}}}$ modulo $\varpi_{L}$ de la série $[b]_{F_{L}, \gamma F_{L}}$ qui est un homomorphisme de groupes formels de $\overline{F_{L}}$ dans $\phi^{\nu_{\gamma}} \overline{F_{L}}$ à coefficients dans $\overline{\mathbb{F}_{q}}$ ne dépendant que de $\nu_{\gamma}$.

La signification de $\{b\}_{\nu_{\gamma}}\left(\varpi_{L_{a b}}\right)$ ou de $\{b\}_{\nu_{\gamma}}\left(\varpi_{\left.L_{i}\right)}\right)$ est claire : pour tout coefficient $\alpha \in \overline{\mathbb{F}}_{q} \subset$ $\mathfrak{N}_{\widetilde{L}}\left(\widetilde{L} L_{a b}\right)$ de $\{b\}_{\nu_{\gamma}}$, la projection canonique de $\alpha$ dans $\widetilde{L}\left(\varpi_{\left.L_{i}\right)}\right)$ est $\widehat{\alpha}^{\left(q_{L}^{i-1}\right)^{-1}}=\phi_{L}^{1-i}(\widehat{\alpha})$ où $\widehat{\alpha}$ désigne le représentant multiplicatif de $\alpha$ dans $\widetilde{L}$, parceque

$$
\mathfrak{N}_{\widetilde{L}}\left(\widetilde{L} L_{a b}\right)^{\times}=\lim _{i \geqslant 1} \widetilde{L}\left(\varpi_{\left.L_{i}\right)}\right)^{\times}
$$

et que, pour tout $i \geqslant 1$, l'extension $\widetilde{L}\left(\varpi_{\left.L_{i}\right)}\right) / \widetilde{L}$ est totalement ramifiée de degré $(q-1) q^{i-1}$ (voir $\S 1.3)$.

Soit $\widetilde{\gamma}=\iota_{L}(\gamma, b) \in \operatorname{Aut}\left(L_{a b}\right)$; on a $\widetilde{\gamma}\left(\varpi_{\left.L_{i}\right)}\right)=\phi^{1-i}[b]_{F, \gamma F}\left(\varpi_{\left.L_{i}\right)}\right)$. Si $S_{i}$ est la série dont les coefficients sont les projections sur $\widetilde{L}\left(\varpi_{\left.L_{i}\right)}\right)$ de ceux de $\{b\}_{\nu_{\gamma}}$, on a $S_{i}=\phi_{L}^{1-i} S_{1}$. Comme les séries $\phi_{L}^{1-i}[b]_{F, \gamma F}$ et $\phi_{L}^{1-i} S_{1}$ sont à coefficients dans $\mathcal{O}(\widetilde{L})$ et coïncident modulo $\varpi_{L}$, on a

$$
\widetilde{\gamma}\left(\varpi_{\left.L_{i}\right)}\right)-S_{i}\left(\varpi_{\left.L_{i}\right)}\right)=\phi^{1-i}[b]_{F, \gamma F}\left(\varpi_{\left.L_{i}\right)}\right)-\phi^{1-i} S_{1}\left(\varpi_{\left.L_{i}\right)}\right) \in \varpi_{\left.L_{i}\right)}^{(q-1) q^{i-1}} \mathcal{O}\left(\widetilde{L}\left(\varpi_{\left.L_{i}\right)}\right) .\right.
$$

De plus, comme $(q-1) q^{i-1}$ est le plus petit nombre de ramification de l'extension $\widetilde{L}_{a b} / \widetilde{L}\left(\varpi_{\left.L_{i}\right)}\right)$, la norme induit alors un homomorphisme d'anneaux de $\mathcal{O}\left(\widetilde{L}\left(\varpi_{\left.L_{i+1}\right)}\right)\right) / \varpi_{\left.L_{i+1}\right)}^{(q-1) q^{i}} \mathcal{O}\left(\widetilde{L}\left(\varpi_{\left.L_{i+1}\right)}\right)\right)$ dans $\mathcal{O}\left(\widetilde{L}\left(\varpi_{\left.L_{i}\right)}\right)\right) / \varpi_{\left.L_{i}\right)}^{(q-1) q^{i-1}} \mathcal{O}\left(\widetilde{L}\left(\varpi_{\left.L_{i}\right)}\right)\right)$ et $\mathcal{O}\left(\mathfrak{N}_{\widetilde{L}}\left(\widetilde{L} L_{a b}\right)\right)$ s'identifie à

$$
\lim _{i \geqslant 1} \mathcal{O}\left(\widetilde{L}\left(\varpi_{\left.L_{i}\right)}\right)\right) / \omega_{i}^{(q-1) q^{i-1}} \mathcal{O}\left(\widetilde{L}\left(\varpi_{\left.L_{i}\right)}\right)\right)
$$

canoniquement (voir [Win83, Section 2.3]). Il en résulte qu'on a bien $\mathfrak{N}_{\widetilde{L} L_{a b} / \widetilde{L}}\left(\iota_{L}(\gamma, b)\right)\left(\varpi_{L_{a b}}\right)=$ $\{b\}_{\nu_{\gamma}}\left(\varpi_{L_{a b}}\right)$, ce qui établit le point (i) de la proposition 2.

Le point (ii) de la proposition 2 en résulte facilement : soit $\bar{\gamma}=\left.\tilde{\gamma}\right|_{M}=\bar{\iota}_{L}(\gamma, b) \in \operatorname{Aut}(M) ; \operatorname{comme}$ $\mathfrak{N}_{\widetilde{L} M / \widetilde{L}}(\bar{\gamma})\left(\varpi_{M}\right)$ est fixe par $\phi_{L}$, on a $\mathfrak{N}_{M / L}(\bar{\gamma})\left(\varpi_{M}\right)=\mathfrak{N}_{\widetilde{L} M / \widetilde{L}}(\bar{\gamma})\left(\varpi_{M}\right)=\widetilde{N}_{L_{a b}^{\left\langle\phi_{L}\right\rangle} / M} \mathfrak{N}_{\widetilde{L}_{L_{a b} / \widetilde{L}}(\tilde{\gamma})}$ $\left(\varpi_{L_{a b}}\right)=(b)_{\bar{\nu}_{\gamma}}\left(\varpi_{M}\right)$ avec $(b)_{\bar{\nu}_{\gamma}}(X)=\vartheta_{L_{a b}^{\left\langle\phi_{L}\right\rangle} / M}\left(\{b\}_{\nu_{\gamma}}(X)\right)$.

\section{Remarques.}

(1) Pour $\gamma=1$, le point (i) est dû à Wintenberger [Win78, Proposition 2.3.2.2].

(2) Compte-tenu des propositions 5 et 6 , la proposition 3 est complètement démontrée.

\section{Une application de réciprocité non abélienne}

\subsection{Une famille d'extensions compatibles de $K$}

Pour tous entiers $n, d \geqslant 1$, on rapelle que $K_{d}$ désigne l'extension non ramifiée de degré $d$ de $K$, on pose $M_{d}^{(0)}=K_{d}$ et on note $M_{d}^{(n)}$ l'extension galoisienne de $K$, résoluble de classe $n$ sur $K_{d}$, invariante par $\phi^{d}$ et maximale pour ces propriétés. 


\section{UNE THÉORIE DU CORPS DE CLASSES LOCAL NON ABÉLIEN}

C'est une extension compatible de $K$; elle est arithmétiquement profinie à cause de la transitivité des corps de normes parce que les extensions abéliennes totalement ramifiées des corps locaux à corps résiduel fini sont arithmétiquement profinies (voir $\S 1.2$ ).

De plus $n^{\prime} \geqslant n, d \mid d^{\prime} \Rightarrow M_{d}^{(n)} \subset M_{d^{\prime}}^{\left(n^{\prime}\right)}$ et $G\left(\mathfrak{g}_{d}^{(n)}, \varpi_{d}^{(n)}\right)$ est le groupe de Koch-de Shalit pour $\mathfrak{g}_{d}^{(n)}=\mathfrak{N}_{K}\left(\operatorname{Gal}\left(M_{d}^{(n)} / K\right)\right)$. On note aussi $M_{d}=\bigcup_{n \geqslant 0} M_{d}^{(n)}, M^{(n)}=\bigcup_{d \geqslant 1} M_{d}^{(n)}, \varpi_{d}^{(n)}=\varpi_{M_{d}^{(n)}}$, $\mathcal{G}_{d}^{(n)}(K, \phi)=\mathcal{G}_{M_{d}^{(n)}}(K, \phi), \iota_{d}^{(n)}=\iota_{M_{d}^{(n)}}$ et $\bar{\iota}_{d}^{(n)}=\bar{\iota}_{M_{d}^{(n)}}$.

Rappelons (voir $\S 1.4)$ que $\mathcal{G}_{d}^{(n)}(K, \phi)$ est un ensemble de couples $(\bar{\nu}, \xi(X)) \in \mathbb{Z} / q^{d} \mathbb{Z} \times X \mathbb{F}_{q^{d}}[[X]]$ qui est un groupe isomorphe à $\operatorname{Gal}\left(M_{d}^{(n)} / K\right)$ pour la loi

$$
(\bar{\nu}, \xi)(\bar{\mu}, \eta)=\left(\bar{\nu}+\bar{\mu}, \phi^{\bar{\nu}} \eta \circ \xi\right) .
$$

Pour $n^{\prime} \geqslant n \geqslant 0, d \mid d^{\prime}$, on note : $F_{d}^{(n)}=F_{M_{d}^{(n)}}$, pour les groupes étiquetés (voir $\S 2.1 .3$ ),

$$
\vartheta_{d^{\prime}, d}^{\left(n^{\prime}, n\right)}=\vartheta_{M_{d^{\prime}}^{\left(n^{\prime}\right)} / M_{d}^{(n)}}, \quad \vartheta_{d}^{\left(n^{\prime}, n\right)}=\vartheta_{d, d}^{\left(n^{\prime}, n\right)}, \quad \vartheta_{d^{\prime}, d}^{(n)}=\vartheta_{d^{\prime}, d}^{(n, n)},
$$

pour les projections canoniques (voir $\S 1.3$ ). Nous avons le diagramme suivant.

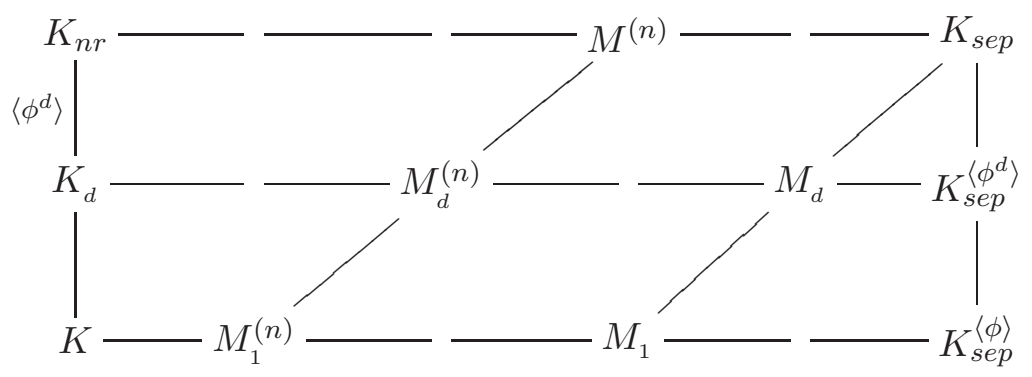

On note également :

$$
L_{d}^{(n+1)}=\left(M_{d}^{(n)}\right)_{a b} \cap K_{s e p}^{\left\langle\phi^{d}\right\rangle} \supset M_{d}^{(n+1)} \quad \text { et } \quad \bar{\vartheta}_{d}^{(n)}=\vartheta_{L_{d}^{(n)} / M_{d}^{(n)}} .
$$

Nous avons le diagramme suivant.

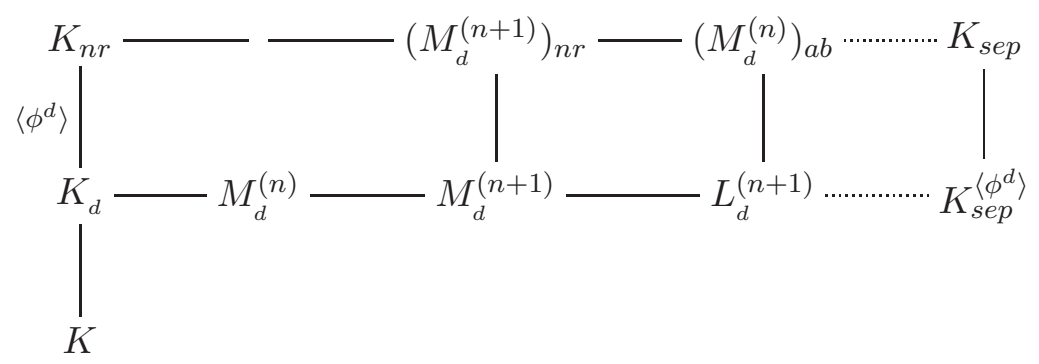

Lemme 5. Pour tout $n, d \geqslant 1$, on a :

(i) $L_{d}^{(1)}=M_{d}^{(1)}$;

(ii) $\bigcup_{d, n \geqslant 1} M_{d}^{(n)}=\bigcup_{d \geqslant 1} M_{d}=K_{\text {sep }}$;

(iii) $M_{d}=\bigcup_{n \geqslant 0} M_{d}^{(n)}$ est l'extension galoisienne maximale de $K$ fixe par $\phi^{d}$;

(iv) $M^{(n)}=\bigcup_{d \geqslant 1} M_{d}^{(n)}$ est l'extension résoluble de classe $n$ maximale de $M^{(0)}=K_{n r}$;

(v) $\left(M_{d}^{(n)}\right)_{a b} \cap M_{d}^{(n+2)}=M_{d}^{(n+1)}$.

Démonstration. Le fait que $L_{d}^{(1)}=M_{d}^{(1)}$ provient du corollaire à la proposition 1 . Soit $x \in K_{\text {sep }}$, il existe $d \geqslant 1$ tel que $\phi^{d}$ laisse fixe $x$ ainsi que tous ses conjugués sur $K$; la clôture galoisienne 


\section{F. LAUBiE}

de $K(x)$ sur $K$ étant résoluble, c'est une sous-extension de $M_{d}^{(n)} / K$ pour tout entier $n$ assez grand, d'où (ii) et (iii) ; et si, de plus, $M^{\left(n_{0}\right)}(x) / M^{\left(n_{0}\right)}$ est abélienne, alors, $M^{\left(n_{0}\right)}(x) / K_{d}$ est résoluble de classe $n_{0}+1$, d'où (iv). Enfin (v) exprime que $M_{d}^{(n+1)} / K$ est la sous-extension galoisienne maximale de $M_{d}^{(n+2)} / K$ qui est abélienne sur $M_{d}^{(n)}$.

\subsection{Description du groupe de Koch-de Shalit en termes de séries formelles}

On se propose de donner une autre description du groupe de Koch-de Shalit absolu

$$
\lim _{\longleftarrow} G\left(\mathfrak{g}_{d}^{(n)}, \varpi_{d}^{(n)}\right):
$$

on décrit un système de groupes $\left\{\mathfrak{G}_{d}^{(n)}(K, \phi)\right\}_{d, n}$ où $\mathfrak{G}_{d}^{(n)}(K, \phi) \simeq G\left(\mathfrak{g}_{d}^{(n)}, \varpi_{d}^{(n)}\right)$ à l'aide de séries formelles et des projections $\vartheta_{L / K}$ définies au $\S 1.3$.

Pour $n=0, \mathfrak{g}_{d}^{(0)}=\operatorname{Gal}\left(K_{d} / K\right), G\left(\mathfrak{g}_{d}^{(0)}, \varpi_{d}^{(0)}\right)=\Gamma_{d}^{(0)} \times U\left(K_{d}\right)$, où $\Gamma_{d}^{(0)}=\operatorname{Gal}\left(K_{n r} / K\right)=\phi^{\widehat{\mathbb{Z}}} ;$ toute unité $u \in U\left(K_{d}\right)$ peut être considérée comme la série $\xi^{(0)}(X) \in \mathbb{F}_{q^{d}}[[X]]$ telle que $\xi^{(0)}\left(\varpi_{d}^{(0)}\right)=u$.

On note $\mathfrak{G}_{d}^{(0)}(K, \phi)=\hat{\mathbb{Z}} \times U\left(K_{d}\right)$ avec la loi :

$$
\left(\nu_{1}, u_{1}\right)\left(\nu_{2}, u_{2}\right)=\left(\nu_{1}+\nu_{2}, u_{1} \phi^{\nu_{1}}\left(u_{2}\right)\right)
$$

Soit $(\nu, u) \in \mathfrak{G}_{d}^{(0)}(K, \phi)$, avec $u=\xi^{(0)}\left(\varpi_{d}^{(0)}\right)$, soit $\bar{\nu}$ la classe de $\nu$ modulo $d$; on rappelle que $[u]_{F_{d}^{(0)}, \phi^{\nu} F_{d}^{(0)}}(X) \in \mathcal{O}\left(K_{d}\right)[[X]]$ est l'isomorphisme de groupes formels, $\equiv u X \bmod X^{2}$, de $F_{d}^{(0)}$ dans

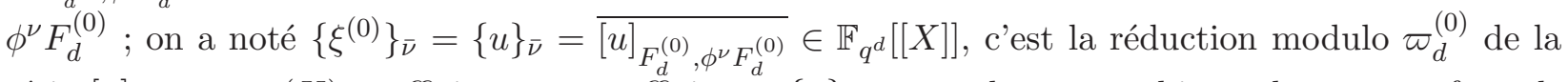
série $[u]_{F_{d}^{(0)}, \phi^{\nu} F_{d}^{(0)}}(X)$ coefficient par coefficient ; $\{u\}_{\bar{\nu}}$ est un homomorphisme de groupes formels de $\bar{F}_{d}^{(0)}$ dans $\phi^{\bar{\nu}} \bar{F}_{d}^{(0)}=\overline{\phi^{\nu} F_{d}^{(0)}}$ et $\bar{\iota}_{d}^{(0)}$ s'identifie à

$$
\begin{gathered}
\mathfrak{G}_{d}^{(0)}(K, \phi) \longrightarrow \mathcal{G}_{d}^{(1)}(K, \phi) \simeq \mathfrak{g}_{d}^{(1)} \\
(\nu, u) \mapsto\left(\bar{\nu},\{u\}_{\bar{\nu}}\right) .
\end{gathered}
$$

Pour $n=1$, définissons $\mathfrak{G}_{d}^{(1)}(K, \phi)$ comme le groupe, isomorphe à $G\left(\mathfrak{g}_{d}^{(1)}, \varpi_{d}^{(1)}\right)$, constitué des $\left(\nu, \xi^{(1)}\right), \nu \in \hat{\mathbb{Z}}, \xi^{(1)}=\xi^{(1)}(X) \in \overline{\mathbb{F}}_{q}[[X]]^{\times},\left(\xi^{(1)}\left(\varpi_{d}^{(1)}\right) \in \overline{\mathbb{F}}_{q}\left(\left(\varpi_{d}^{(1)}\right)\right) \simeq \mathfrak{N}_{\widetilde{K}}\left(\widetilde{K} M_{d}^{(1)}\right)\right)$ tels que $\vartheta_{d}^{(1,0)}\left(\xi^{(1)}\right)=\xi^{(0)}, \xi^{(0)}\left(\varpi_{1}^{(0)}\right)=u \in U\left(K_{d}\right)$, et $\phi^{d} \xi^{(1)}(X) / \xi^{(1)}(X)=\left\{\xi^{(0)}\right\}_{\bar{\nu}}(X) / X$, pour la loi

$$
\left(\nu_{1}, \xi_{1}^{(1)}\right)\left(\nu_{2}, \xi_{2}^{(1)}\right)=\left(\nu_{1}+\nu_{2}, \xi_{1}^{(1)} \cdot \phi^{\nu_{1}} \xi_{2}^{(1)} \circ\left\{\xi_{1}^{(0)}\right\}_{\bar{\nu}_{1}}\right) \text {; }
$$

l'ordre des opérations est $\xi_{1}^{(1)} \cdot\left(\left(\phi^{\nu_{1}} \xi_{2}^{(1)}\right) \circ\left\{\xi_{1}^{(0)}\right\}_{\bar{\nu}_{1}}\right)$.

Soit $\gamma_{d}^{(1)}=\left(\bar{\nu},\{u\}_{\bar{\nu}}\right) \in \mathcal{G}_{d}^{(1)}(K, \phi) \simeq \mathfrak{g}_{d}^{(1)} ;$ l'isomorphisme de groupes formels $\left[\xi^{(1)}\left(\varpi_{d}^{(1)}\right)\right]_{F_{d}^{(1)}, \gamma_{d}^{(1)} F_{d}^{(1)}}(X) \in \overline{\mathbb{F}}_{q}\left[\left[\varpi_{d}^{(1)}, X\right]\right]$ est une série à coefficients dans $\overline{\mathbb{F}}_{q}\left[\left[\varpi_{d}^{(1)}\right]\right]$ dont la réduction modulo $\varpi_{d}^{(1)}$, notée $\left\{\xi^{(1)}\right\}_{\nu}$ appartient à $\overline{\mathbb{F}}_{q}[[X]]$. Soit $\left(\xi^{(1)}\right)_{\bar{\nu}}=\bar{\vartheta}_{d}^{(1)}\left(\left\{\xi^{(1)}\right\}_{\nu}\right) \in \mathbb{F}_{q^{d}}[[X]] ;$ alors $\bar{\iota}_{d}^{(1)}$ s'identifie à

$$
\begin{gathered}
\mathfrak{G}_{d}^{(1)}(K, \phi) \longrightarrow \mathcal{G}_{d}^{(2)}(K, \phi) \simeq \mathfrak{g}_{d}^{(2)} \\
\left(\nu, \xi^{(1)}\right) \mapsto\left(\bar{\nu},\left(\xi^{(1)}\right)_{\bar{\nu}}\right) .
\end{gathered}
$$

Pour $n \geqslant 2$, on définit par récurrence $\mathfrak{G}_{d}^{(n)}(K, \phi)$ comme le groupe, isomorphe à $G\left(\mathfrak{g}_{d}^{(n)}, \varpi_{d}^{(n)}\right)$, constitué des $\left(\nu, \xi^{(n)}\right), \nu \in \hat{\mathbb{Z}}, \xi^{(n)}=\xi^{(n)}(X) \in \overline{\mathbb{F}}_{q}[[X]]^{\times},\left(\xi^{(1)}\left(\varpi_{d}^{(n)}\right) \in \overline{\mathbb{F}}_{q}\left(\left(\varpi_{d}^{(n)}\right)\right) \simeq \mathfrak{N}_{\widetilde{K}}\left(\widetilde{K} M_{d}^{(n)}\right)\right)$ tels que $\vartheta_{d}^{(n, n-1)}\left(\xi^{(n)}\right)=\xi^{(n-1)}$ avec $\left(\nu, \xi^{(n-1)}\right) \in \mathfrak{G}_{d}^{(n-1)}(K, \phi)$ et $\phi^{d} \xi^{(n)}(X) / \xi^{(n)}(X)=\left(\xi^{(n-1)}\right)_{\bar{\nu}}(X) / X$, $\left(\xi^{(n-1)}\right)_{\bar{\nu}}=\bar{\vartheta}_{d}^{(n-1)}\left(\left\{\xi^{(n-1)}\right\}_{\bar{\nu}}\right)$ pour la loi

$$
\left(\nu_{1}, \xi_{1}^{(n)}\right)\left(\nu_{2}, \xi_{2}^{(n)}\right)=\left(\nu_{1}+\nu_{2}, \xi_{1}^{(n)} \cdot \phi^{\nu_{1}} \xi_{2}^{(n)} \circ\left(\xi_{1}^{(n-1)}\right)_{\bar{\nu}}\right) .
$$


UNE THÉORIE DU CORPS DE CLASSES LOCAL NON ABÉLIEN

Soit $\gamma_{d}^{(n)}=\left(\bar{\nu},\left(\xi^{(n-1)}\right)_{\bar{\nu}}\right) \in \mathcal{G}_{d}^{(n)}(K, \phi) ;$ l'isomorphisme de groupes formels $\left[\xi^{(n)}\left(\varpi_{d}^{(n)}\right)\right]_{F_{d}^{(n)}, \gamma_{d}^{(n)} F_{d}^{(n)}}$ $(X) \in \overline{\mathbb{F}}_{q}\left[\left[\varpi_{d}^{(n)}, X\right]\right]$ est une série à coefficients dans $\overline{\mathbb{F}}_{q}\left[\left[\varpi_{d}^{(n)}\right]\right]$ dont la réduction modulo $\varpi_{d}^{(n)}$, notée $\left\{\xi^{(n)}\right\}_{\nu}$ appartient à $\overline{\mathbb{F}}_{q}[[X]]$. Soit $\left(\xi^{(n)}\right)_{\bar{\nu}}=\bar{\vartheta}_{d}^{(n)}\left(\left\{\xi^{(n)}\right\}_{\nu}\right) \in \mathbb{F}_{q^{d}}[[X]]$ alors $\bar{\iota}_{d}^{(n)}$ s'identifie à

$$
\begin{gathered}
\mathfrak{G}_{d}^{(n)}(K, \phi) \longrightarrow \mathcal{G}_{d}^{(n+1)}(K, \phi) \simeq \mathfrak{g}_{d}^{(n+1)} \\
\left(\nu, \xi^{(n)}\right) \mapsto\left(\bar{\nu},\left(\xi^{(n)}\right)_{\bar{\nu}}\right) .
\end{gathered}
$$

Pour tous $n \geqslant m \geqslant 0$ et $e \geqslant 1$, on a des homomorphismes de transition surjectifs :

$$
\begin{aligned}
& \mathfrak{G}_{d e}^{(n)}(K, \phi) \rightarrow \mathfrak{G}_{d}^{(m)}(K, \phi) \quad \mathcal{G}_{d e}^{(n+1)}(K, \phi) \rightarrow \mathcal{G}_{d}^{(m+1)}(K, \phi) \\
& \left(\nu, \xi^{(n)}\right) \mapsto\left(\nu, \prod_{0 \leqslant i<e} \phi^{d i}\left(\vartheta_{d e, d}^{(n, m)}\left(\xi^{(n)}\right)\right)\right) \quad\left(\bar{\nu},\left(\xi^{(n)}\right)_{\bar{\nu}}\right) \mapsto\left(\overline{\bar{\nu}}, \vartheta_{d e, d}^{(n+1, m+1)}\left(\left(\xi^{(n)}\right)_{\bar{\nu}}\right)\right)
\end{aligned}
$$

( $\overline{\bar{\nu}}$ désigne la classe modulo $d$ de $\bar{\nu} \in \mathbb{Z} /$ de $\mathbb{Z}$ ), qui permettent de définir les groupes

$$
\begin{aligned}
& \mathfrak{G}_{d}(K, \phi)={\underset{\lim }{n}}_{\mathfrak{G}_{d}^{(n)}}(K, \phi), \quad \mathcal{G}_{d}(K, \phi)=\overleftarrow{\lim }_{n} \mathcal{G}_{d}^{(n)}(K, \phi), \\
& \mathfrak{G}^{(n)}(K, \phi)=\underbrace{\lim }_{d} \mathfrak{G}_{d}^{(n)}(K, \phi), \quad \mathcal{G}^{(n)}(K, \phi)=\underbrace{\lim }_{d} \mathcal{G}_{d}^{(n)}(K, \phi), \\
& \mathfrak{G}(K, \phi)=\lim _{n, d} \mathfrak{G}_{d}^{(n)}(K, \phi) \quad \text { et } \quad \mathcal{G}(K, \phi)={\underset{n}{n, d}}_{\lim } \mathcal{G}_{d}^{(n)}(K, \phi),
\end{aligned}
$$

(il est clair que $\mathcal{G}(K, \phi)$ est bien le groupe défini au $\S 1.4)$, et un isomorphisme de groupes dépendant de $\phi$ :

$$
\begin{gathered}
\Re_{K}: \mathfrak{G}(K, \phi) \longrightarrow \mathcal{G}(K, \phi) \\
\left(\left(\nu, \xi_{d}^{(n)}\right)\right)_{n, d} \mapsto\left(\left(\bar{\nu},\left(\xi_{d}^{(n)}\right)_{\bar{\nu}}\right)\right)_{n, d}
\end{gathered}
$$

qui applique (non injectivement) $\mathfrak{G}_{d}(K, \phi)$ sur $\mathcal{G}_{d}(K, \phi)$ et $\mathfrak{G}^{(n)}(K, \phi)$ sur $\mathcal{G}^{(n)}(K, \phi)$.

\subsection{Topologies et ramification}

Comme on l'a déjà dit, les questions liées à la ramification feront ultérieurement l'objet d'une étude plus détaillée.

Cependant les filtrations de ramification des groupes quotients de $\mathcal{G}(K, \phi)$ correspondant aux extensions arithmétiquement profinies de $K$ peuvent être facilement décrites grâce à la théorie du corps de normes.

Soit $L$ une extension arithmétiquement profinie, galoisienne et compatible de $K$, soit $d$ son degré résiduel et soit $\mathcal{G}_{L}(K, \phi)$ le quotient de $\mathcal{G}(K, \phi)$ isomorphe à $\operatorname{Gal}(L / K)$. On rappelle (voir $\S 1.4)$ qu'un $K$-automorphisme $\sigma$ de $L$ s'identifie au couple $\left(\nu_{\sigma}, \sigma_{L}(X)\right) \in \mathbb{Z} / d \mathbb{Z} \times X \mathbb{F}_{q^{d}}[[X]]$ tel que

$$
\mathfrak{N}_{L / K}(\sigma)\left(\sum_{i} \alpha_{i} \varpi_{L}^{i}\right)=\sum_{i} \phi^{\nu_{\sigma}}\left(\alpha_{i}\right) \sigma_{L}\left(\varpi_{L}\right)^{i}
$$

pour tout $\sum_{i} \alpha_{i} \varpi_{L}^{i} \in \mathbb{F}_{q^{d}}\left(\left(\varpi_{L}\right)\right)$.

Comme $L / K$ est arithmétiquement profinie, la filtration de ramification de $\operatorname{Gal}(L / K)$ est bien définie en numérotations supérieure et inférieure [Win83, Section 1.1] ; la filtration de ramification de $\mathfrak{N}_{K}(\operatorname{Gal}(L / K))$ est donnée a priori en numérotation inférieure par sa fonction d'ordre $i_{\mathfrak{N}_{K}(L)}$ définie par :

$$
i_{\mathfrak{N}_{K}(L)}\left(\mathfrak{N}_{L / K}(\sigma)\right)= \begin{cases}-1 & \text { si } \nu_{\sigma} \neq 0 \\ v_{\mathfrak{N}_{K}(L)}\left(\sigma_{L}\left(\varpi_{L}\right) / \varpi_{L}-1\right) & \text { sinon }\end{cases}
$$

où $v_{\mathfrak{N}_{K}(L)}$ est la valuation normalisée par $v_{\mathfrak{N}_{K}(L)}\left(\varpi_{L}\right)=1$. 


\section{F. LAUBiE}

En outre l'application $\sigma \mapsto \mathfrak{N}_{L / K}(\sigma)$ respecte les filtrations de ramification [Win83, Section 3.3]. Pour tout $i \geqslant 0$, posons

$$
\mathcal{G}_{d}^{(n)}(K, \phi)_{i}=\left\{(0, \xi(X)) \in \mathcal{G}_{d}^{(n)}(K, \phi) ; \xi(X) \equiv X \bmod X^{i+1}\right\}
$$

c'est l'image par $\mathfrak{N}_{L / K}(\sigma)$ du groupe de ramification $\operatorname{Gal}\left(M_{d}^{(n+1)} / K\right)_{i}$.

La numérotation supérieure de la ramification de $\mathcal{G}_{d}^{(n)}(K, \phi)$ est définie par :

$$
\mathcal{G}_{d}^{(n)}(K, \phi)^{u}=\mathcal{G}_{d}^{(n)}(K, \phi)_{i} \quad \text { avec } u=\int_{0}^{i} \frac{d t}{\left(\mathcal{G}_{d}^{(n)}(K, \phi)_{0}: \mathcal{G}_{d}^{(n)}(K, \phi)_{t}\right)}
$$

et celle de $\mathcal{G}(K, \phi)$ par

$$
\mathcal{G}(K, \phi)^{u}=\lim _{n, d} \mathcal{G}_{d}^{(n)}(K, \phi)^{u} .
$$

On est donc amené à définir $\mathfrak{G}(K, \phi)^{u}=\Re_{K}^{-1}\left(\left(\mathcal{G}(K, \phi)^{u}\right)\right.$ et la question qui se pose naturellement est de décrire les sous-groupes $\mathfrak{G}(K, \phi)^{u}$.

La topologie sur $\mathfrak{G}(K, \phi)$ pour laquelle $\left\{\mathfrak{G}(K, \phi)^{u}\right\}_{u \in \mathbb{R}^{+}}$est une base de voisinages de 1 est complète et pour cette topologie, $\Re_{K}$ est continu.

\subsection{Norme et changement du corps de base}

Pour toute extension finie compatible $K^{\prime} / K$, de degré résiduel $f$, on définit $\mathfrak{G}\left(K^{\prime}, \phi^{\prime}\right)$ et $\mathcal{G}\left(K^{\prime}, \phi^{\prime}\right)$ à l'aide du dévissage de Lubin-Tate $\left(K^{\prime}, \phi^{\prime}\right)$ canoniquement déduit de $(K, \phi)$ conformément à $\S 2.2 .5$.

Soit $\mathcal{R}\left(K^{\prime}\right)$ l'ensembles des extensions de $K$ galoisiennes, résolubles de classe finie, compatibles et contenant $K^{\prime}$. Tout $L \in \mathcal{R}\left(K^{\prime}\right)$ est une extension arithmétiquement profinie de $K$ et $K_{\text {sep }}=$ $\bigcup_{L \in \mathcal{R}\left(K^{\prime}\right)} L$. On note $\jmath_{K^{\prime}}$ la bijection

$$
\begin{gathered}
G_{K^{\prime}} \stackrel{\jmath_{K^{\prime}}}{\longrightarrow} \mathcal{G}\left(K^{\prime}, \phi^{\prime}\right) \\
\sigma \mapsto\left(\nu,\left(\sigma_{L}(X)\right)_{L \in \mathcal{R}\left(K^{\prime}\right)}\right) .
\end{gathered}
$$

On munit $\mathcal{G}\left(K^{\prime}, \phi^{\prime}\right)$ de la structure de groupe compact pour laquelle $\jmath_{K^{\prime}}$ est un isomorphisme de groupes topologiques.

On note $\mathcal{N}(L / K)=\jmath_{K}\left(G_{L}\right)$, pour toute extension séparable $L / K$.

En outre, $\mathcal{R}\left(K^{\prime}\right)$ est une partie cofinale de $\mathcal{R}(K)$ pour l'inclusion, il y a donc une application canonique

$$
\begin{gathered}
\mathcal{N}_{K^{\prime} / K} \mathcal{G}\left(K^{\prime}, \phi^{\prime}\right) \longrightarrow \mathcal{G}(K, \phi) \\
\left(\nu,\left(\sigma_{L}(X)\right)_{L \in \mathcal{R}\left(K^{\prime}\right)}\right) \mapsto\left(f \nu,\left(\sigma_{L}(X)\right)_{L \in \mathcal{R}\left(K^{\prime}\right)}\right) .
\end{gathered}
$$

C'est un homomorphisme injectif et continu de groupes et $\mathcal{N}_{K^{\prime} / K} \circ \mathcal{N}_{K^{\prime \prime}} / K^{\prime}=\mathcal{N}_{K^{\prime \prime} / K}$ pour $K^{\prime \prime} \supset$ $K^{\prime} \supset K$, compatibles.

D'après $\S 1.4$, on a :

$$
\mathcal{N}\left(K^{\prime} / K\right)=\mathcal{N}_{K^{\prime} / K}\left(\mathcal{G}\left(K^{\prime}, \phi^{\prime}\right)\right)
$$

et c'est un sous-groupe fermé d'indice fini de $\mathcal{G}(K, \phi)$ isomorphe à $G_{K^{\prime}}$.

Soit

$$
\begin{gathered}
\mathfrak{n}_{K^{\prime} / K}: \mathfrak{G}\left(K^{\prime}, \phi^{\prime}\right) \rightarrow \mathfrak{G}(K, \phi) \\
\left(\left(\nu, \xi_{d}^{\prime(n)}\right)\right)_{n, d} \mapsto\left(\left(f \nu, \xi_{d}^{(n)}\right)\right)_{n, d}
\end{gathered}
$$


où

$$
\xi_{d}^{(n)}=\prod_{0 \leqslant i<f-1} \phi^{d i}\left(\vartheta_{M_{d}^{\prime(n)} / M_{d}^{(n)}}\left(\xi_{d}^{\prime(n)}\right)\right) .
$$

C'est un homomorphisme de groupes injectif ; on peut montrer que $\mathfrak{n}_{K^{\prime} / K}$ généralise la norme métabélienne de Koch et de Shalit [KdS96, Section 1.5] ; elle satisfait les relations de transitivité attendues $\mathfrak{n}_{K^{\prime} / K} \circ \mathfrak{n}_{K^{\prime \prime} / K^{\prime}}=\mathfrak{n}_{K^{\prime \prime} / K}$ pour $K^{\prime \prime} \supset K^{\prime} \supset K$, compatibles.

On définit

$$
\mathfrak{n}\left(K^{\prime} / K\right)=\mathfrak{n}_{K^{\prime} / K}\left(\mathfrak{G}\left(K^{\prime}, \phi^{\prime}\right)\right) .
$$

C'est un sous-groupe fermé d'indice fini de $\mathfrak{G}(K, \phi)$. Si $K^{\prime} / K$ est infini et réunion d'extensions finies compatibles, on définit $\mathfrak{n}\left(K^{\prime} / K\right)$ comme l'intersection des sous-groupes $\mathfrak{n}(F / K)$ où $F / K$ parcourt l'ensemble de toutes les sous-extensions finies et compatibles de $K^{\prime} / K$; d'après $\S 3.2$, on a

$$
\Re_{K}\left(\mathfrak{n}\left(K^{\prime} / K\right)\right)=\mathcal{N}\left(K^{\prime} / K\right)
$$

et on peut prolonger la définition des sous-groupes $\mathfrak{n}\left(K^{\prime} / K\right)$ à une quelconque extension séparable de $K$ par :

$$
\mathfrak{n}(L / K)=\Re_{K}^{-1}(\mathcal{N}(L / K))
$$

\subsection{Un théorème du corps de classes non abélien}

Pour tout corps $E$ et tout entier $n \geqslant 1$, on note $E_{a b^{n+1}}=\left(E_{a b^{n}}\right)_{a b}$.

ThÉorème. Soit $(K, \phi)$ un dévissage de Lubin-Tate.

(i) Existence. La correspondance $L / K \mapsto \mathfrak{n}(L / K)$, a priori définie uniquement sur les extensions compatibles $L / K$, peut être prolongée en un isomorphisme d'ensembles ordonnés de l'ensemble des extensions algébriques séparables de $K$ sur celui des sous-groupes fermés de $\mathfrak{G}(K, \phi)$. Le sous-groupe $\mathfrak{n}(L / K)$ est d'indice fini si et seulement si $L / K$ est finie, et dans ce cas on a :

$$
[L: K]=[\mathfrak{G}(K, \phi): \mathfrak{n}(L / K)]=[\mathcal{G}(K, \phi): \mathcal{N}(L / K)] .
$$

(ii) L'application de réciprocité. Pour toute extension algébrique séparable $L / K, \Re_{K}$ se factorise en un isomorphisme $\Re_{L / K}$ de $\mathfrak{G}(K, \phi) / \mathfrak{n}(L / K)$ sur $\mathcal{G}(K, \phi) / \mathcal{N}(L / K)$. Après identification de $\operatorname{Gal}(L / K)$ à $\mathcal{G}(K, \phi) / \mathcal{N}(L / K)$, la famille des $\Re_{L / K}$ induit des isomorphismes entre $\mathfrak{G}^{(n)}(K, \phi)$ et $\operatorname{Gal}\left(\left(K_{n r}\right)_{a b^{n+1}} / K\right)$, entre $\mathfrak{G}_{d}(K, \phi)$ et $\operatorname{Gal}\left(\left(M_{d}\right)_{a b} / K\right)$ où $M_{d}$ est l'extension galoisienne maximale de $K$ fixe par $\phi^{d}$ et entre $\mathfrak{G}_{d}^{(n)}(K, \phi)$ et $\operatorname{Gal}\left(\left(M_{d}^{(n)}\right)_{a b} / K\right)$ où $M_{d}^{(n)}$ désigne l'extension galoisienne sur $K$, résoluble de classe $n$ sur $K_{d}$, fixe par $\phi^{d}$ et maximale pour ces propriétés.

(iii) Fonctorialité. Soit $K^{\prime}$ une extension algébrique séparable et compatible de $K$, alors le diagramme suivant commute.

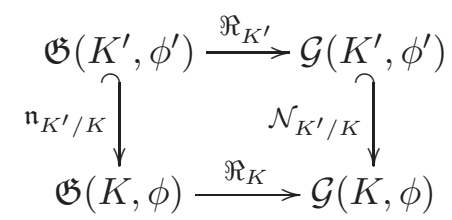

(iv) Relation avec la théorie du corps de classe local abélien. Le noyau de la projection canonique de $\mathfrak{G}(K, \phi)$ sur $\mathfrak{G}_{1}^{(0)}(K, \phi)$ est $\mathfrak{n}\left(K_{a b} / K\right)$. Soit $L / K$ une extension galoisienne ; son groupe des normes universelles s'identifie à $\mathfrak{n}(L / K) \mathfrak{n}\left(K_{a b} / K\right) / \mathfrak{n}\left(K_{a b} / K\right)$ et l'extension abélienne qui est corps de classes pour $\mathfrak{n}(L / K) \mathfrak{n}\left(K_{a b} / K\right) / \mathfrak{n}\left(K_{a b} / K\right)$ est la sous-extension abélienne maximale de $L / K$.

$\left(\mathrm{iv}^{\prime}\right)$ Relation avec la théorie du corps de classe local métabélien. Le noyau de la projection canonique de $\mathfrak{G}(K, \phi)$ sur $\mathfrak{G}_{1}^{(1)}(K, \phi)$ est $\mathfrak{n}\left(K_{a b^{2}} / K\right)$. Soit $L / K$ une extension galoisienne et 


\section{F. LAUBiE}

compatible; alors son groupe de norme métabélienne au sens de Koch et de Shalit [KdS96, Définition 0.4.8] s'identifie à $\mathfrak{n}(L / K) \mathfrak{n}\left(K_{a b^{2}} / K\right) / \mathfrak{n}\left(K_{a b^{2}} / K\right)$ et le corps de classes métabélien pour $\mathfrak{n}(L / K) \mathfrak{n}\left(K_{a b^{2}} / K\right) / \mathfrak{n}\left(K_{a b^{2}} / K\right)$ (c'est à dire l'extension correspondante par l'application de réciprocité métabélienne de Koch et de Shalit) est la sous-extension métabélienne maximale de $L / K$.

(v) Calcul récursif de l'application de réciprocité. Soit $L / K$ une extension galoisienne et compatible, de degré résiduel $f$; on note $L^{(0)} / K$ la sous-extension non ramifiée maximale de $L / K$, $L^{(1)} / L^{(0)}$ la sous-extension abélienne maximale de $L / L^{(0)}$ et, par récurrence, $L^{(n+1)} / L^{(n)}$ la sous-extension abélienne maximale de $L / L^{(n)}$; les extensions $L^{(n)} / K$ sont galoisiennes et compatibles.

On suppose que pour tout $n$, l'extension $L^{(n)} / K$ est finie.

Soit $v=\left(\left(\nu, \xi_{d f}^{(n)}\right)\right)_{n, d} \in \mathfrak{G}(K, \phi)$ et $\sigma$ l'automorphisme de $K_{\text {sep }}$ défini par $\Re_{K}(v)$.

Alors $\xi_{f}^{(0)}\left(\varpi_{K}\right)$ est une unité $u^{(0)}$ de $L^{(0)}=K_{f}$ et $\left.\sigma\right|_{L^{(1)}}$ se prolonge à $L_{a b}^{(0)}$ en l'automorphisme dont la restriction à $K_{n r}$ est $\phi^{\nu}$ et qui applique l'étiquette $\varpi_{L_{i)}^{(0)}} \operatorname{sur} \phi^{1-i}\left[u^{(0)}\right]_{F_{L^{(0)}}, \sigma F_{L^{(0)}}}\left(\varpi_{L_{i)}^{(0)}}\right)$, pour tout $i \geqslant 1$; pour $n \geqslant 1$, la projection canonique $\widetilde{N}_{M_{f}^{(n)} / L^{(n)}}$ de $\mathfrak{N}_{\widetilde{K}}\left(\widetilde{K} M_{f}^{(n)}\right)$ dans $\widetilde{L^{(n)}}$ applique $\varpi_{f}^{(n)} \operatorname{sur} \varpi_{L^{(n)}}$ et $\xi_{f}^{(n)}\left(\varpi_{f}^{(n)}\right)$ sur une unité $u^{(n)}$ de $\widetilde{L^{(n)}}$ telle que $\left.\phi^{f}\left(u^{(n)}\right) / u^{(n)}=\sigma\left(\varpi_{L^{(n)}}\right) / \varpi_{L^{(n)}}\right)$; en outre, $\left.\sigma\right|_{L^{(n+1)}}$ se prolonge en l'automorphisme de $L_{a b}^{(n)}$ dont la restriction à $L_{n r}^{(n)}$ est $\phi^{\nu}$ et qui applique l'étiquette $\varpi_{L_{i)}^{(n)}} \operatorname{sur} \phi^{1-i}\left[u^{(n)}\right]_{F_{L^{(n)}}, \sigma F_{L^{(n)}}}\left(\varpi_{L_{i)}^{(n)}}\right)$, pour tout $i \geqslant 1$.

Commentaires. Les points (i) à (iv) sont déjà complètement établis. Le point (iv') exprime que la construction de Koch et de Shalit pour les extensions métabéliennes est essentiellement la même que la notre ; les détails sont laissés au lecteur.

En ce qui concerne le point (v), rappelons d'abord que la notation $E_{i \text { ) }}$ où $E / K$ est une extension finie compatible est donnée au $\S 2.1 .3$. Détaillons, pour $n \geqslant 1$, la projection canonique :

$$
\begin{gathered}
\mathfrak{G}_{f}^{(n)}(K, \phi) \longrightarrow G\left(\operatorname{Gal}\left(L^{(n)} / K\right), \varpi_{L^{(n)}}\right) \\
\left(\nu, \xi_{f}^{(n)}\right) \mapsto\left(\sigma_{n}, u^{(n)}\right)
\end{gathered}
$$

$\sigma$ est l'automorphisme de $\widetilde{K_{\text {sep }}}$ défini par $\Re_{K}\left(\left(\nu, \xi_{f}^{(n)}\right)\right), \sigma_{n}$ est la restriction de $\sigma$ à $\widetilde{L^{(n)}}$,

$$
u^{(n)}=\vartheta_{M_{f}^{(n)} / L^{(n)}}\left(\xi_{f}^{(n)}\right)\left(\varpi_{L^{(n)}}\right) \in U\left(\widetilde{L^{(n)}}\right) .
$$

Soit $\left(\mu, \eta_{f}^{(n)}\right)$ un autre élément de $\mathfrak{G}_{f}^{(n)}(K, \phi)$ d'image $\left(\tau_{n}, v^{(n)}\right)$ dans $G\left(\operatorname{Gal}\left(L^{(n)} / K\right), \varpi_{L^{(n)}}\right)$; leur produit $\left(\nu+\mu, \xi_{f}^{(n)} \cdot \phi^{\nu} \eta_{f}^{(n)} \circ\left(\xi_{f}^{(n-1)}\right)_{\bar{\nu}}\right)$ s'applique sur $\left(\sigma_{n} \circ \tau_{n}, u^{(n)} \cdot \sigma_{n}\left(v^{(n)}\right)\right)$.

Identifions $u^{(n)}$ à la série $\in \overline{\mathbb{F}}_{q}[[X]]^{\times}$telle que $u^{(n)}\left(\varpi_{L^{(n)}}\right)=u^{(n)}$ et $\sigma_{n}$ à la série $\sum_{i} s_{i} X^{i} \in$ $X \mathbb{F}_{q^{f}}[[X]]$ telle que $\sigma\left(\varpi_{L^{(n)}}\right)=\sum_{i} s_{i} \varpi_{L^{(n)}}^{i}$ et pareil pour $v^{(n)}$ et $\tau_{n}$; alors $\sigma_{n}\left(v^{(n)}\right)$ s'identifie à la série $\phi^{\nu} v^{(n)} \circ \sigma_{n}$ mais $u^{(n)} \cdot \sigma_{n}\left(v^{(n)}\right)$ n'est effectivement le produit des deux séries $u^{(n)}$ et $\phi^{\nu} v^{(n)} \circ \sigma_{n}$ que dans le cas où $K$ est de caractéristique $p$.

\subsection{Commentaires sur la théorie de Gurevich}

Dans sa thèse [Gur98], Gurevich a été le premier à prolonger la théorie de Koch-de Shalit aux extensions infinies résolubles de classe finie, des corps locaux. Comme ci-dessus, la théorie de Gurevich est fondée sur l'image de l'application de réciprocité de Koch et de Shalit dans certains corps de normes. Cependant Gurevich utilise une construction de tours d'extensions de corps locaux différentes de celles du $\S 3.1$ ci-dessus : à la place des corps $M_{d}^{(n)}$, il considère les corps $M_{n}^{\diamond}$ avec la modification essentielle suivante : $M_{n+1}^{\diamond}$ est simplement l'extension abélienne maximale de $M_{n}^{\diamond}$ fixe par $\phi$, alors 


\section{UNE THÉORIE DU CORPS DE CLASSES LOCAL NON ABÉLIEN}

que, pour construire $M_{d}^{(n+1)}$ à partir de $M_{d}^{(n)}$, on considérait la sous-extension maximale galoisienne sur $K$ de l'extension abelienne maximale de $M_{d}^{(n)}$ fixe par $\phi^{d}$.

Il en résulte que, contrairement aux extensions $M_{d}^{(n)} / K$, les extensions $M_{n}^{\diamond} / K$ ne sont pas galoisiennes, pour $n \geqslant 2$. Cependant Gurevich démontre que l'extension non ramifiée maximale de $M_{n}^{\diamond}$ est galoisienne sur $K$.

Cela est suffisant pour appliquer de façon itérative les applications de réciprocité de Koch et de Shalit aux corps de normes correspondant aux $M_{n}^{\diamond}$ mais seulement après extension des corps résiduels à la clôture agébrique de $\mathbb{F}_{p}$. Il en résulte que l'expression de l'application de réciprocité générale se complique par l'obligation de décrire précisément comment se distribue la partie non ramifiée de l'extension dans sa décomposition en sous-extensions abéliennes successives ; en outre les propriétés de fonctorialité de l'application de réciprocité de Gurevich, qui existent certainement, semblent plus délicates à établir et à exprimer, ce qui fait obstacle au passage à la limite en vue d'une description du groupe de Galois absolu. En revanche les calculs de ramification sont plus faciles à mettre en œuvre dans le cadre de la théorie de Gurevich que dans la notre.

\subsection{Sur l'application de réciprocité de Fesenko}

Dans cette section, nous étudions plus particulièrement les extensions fixes par $\phi$.

Rappelons que, pour tout entier $n \geqslant 1$, le groupe $\mathfrak{G}_{1}^{(n)}(K, \phi)$ s'identifie canoniquement à $G\left(\mathfrak{g}_{1}^{(n)}, \varpi_{1}^{(n)}\right)$ de la façon suivante : notons $\Re_{1}^{(n)}=\Re_{\left(M_{1}^{(n)}\right)_{a b} / K}$ l'application de réciprocité définie $\operatorname{sur} \mathfrak{G}_{1}^{(n)}(K, \phi)=\mathfrak{G}(K, \phi) / \mathfrak{n}\left(\left(M_{1}^{(n)}\right)_{a b} / K\right) ;$ soit $\left(\nu, \xi^{(n)}\right) \in \mathfrak{G}_{1}^{(n)}(K, \phi)$ (avec $\nu \in \hat{\mathbb{Z}}$ et $\xi^{(n)}(X) \in$ $\left.\overline{\mathbb{F}}_{q}[[X]]^{\times}\right)$; on considère $\Re_{1}^{(n)}\left(\left(\nu, \xi^{(n)}\right)\right)=\left(0,\left(\xi^{(n-1)}\right)_{0}\right) \in \mathfrak{g}_{1}^{(n)}$ comme un automorphisme de $\mathfrak{M}_{1}^{(n)}=$ $\mathfrak{N}_{K}\left(M_{1}^{(n)}\right)$ qu'on relève en l'automorphisme $\gamma^{(n)}$ de $\widetilde{\mathfrak{M}}_{1}^{(n)}$ dont la restriction au corps résiduel $\overline{\mathbb{F}}_{q}$ est $\phi^{\nu}$; d'autre part $\xi^{(n)}\left(\varpi_{1}^{(n)}\right)$ est une unité $b^{(n)}$ de $\widetilde{\mathfrak{M}}_{1}^{(n)}$; alors $\left(\gamma^{(n)}, b^{(n)}\right)$ est bien l'élément de $G\left(\mathfrak{g}_{1}^{(n)}, \varpi_{1}^{(n)}\right)$ associé à $\left(\nu, \xi^{(n)}\right) \in \mathfrak{g}_{1}^{(n)}$.

Dans cette identification, l'application de réciprocité $\Re_{1}^{(n)}$ est la flèche diagonale du diagramme commutatif suivant.

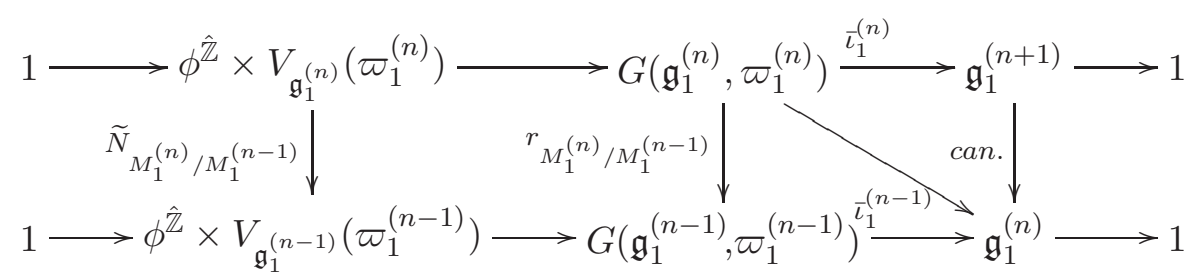

Son noyau est le produit direct de groupes $\phi^{\hat{\mathbb{Z}}} \times U_{1}^{(n)}$ où $U_{1}^{(n)}=U\left(M_{1}^{(n)}\right)$ car la restriction de $\bar{\iota}_{1}^{(n)}$ à ce sous-groupe est l'inverse de l'application de réciprocité d'Artin $\left(*,\left(\mathfrak{M}_{1}^{(n)}\right)_{a b} / \mathfrak{M}_{1}^{(n)}\right)$; en outre $G\left(\mathfrak{g}_{1}^{(n)}, \varpi_{1}^{(n)}\right) /\left(\phi^{\hat{\mathbb{Z}}} \times U_{1}^{(n)}\right)$ s'identifie au quotient du groupe $\mathcal{U}_{1}^{(n)}=\left\{b=b_{\sigma} \in U\left(\widetilde{\mathfrak{M}}_{1}^{(n)}\right) ; \exists \sigma \in\right.$ $\left.G_{K}, \phi(b) / b=\sigma\left(\varpi_{1}^{(n)}\right) / \varpi_{1}^{(n)}\right\}$ pour la loi $b_{\sigma} \star b_{\tau}=b_{\sigma} \cdot \sigma\left(b_{\tau}\right)$ par son sous-groupe $U_{1}^{(n)}$.

Cela signifie que l'application qui à $\sigma \in \mathfrak{g}_{1}^{(n)}$ associe la classe $v_{\sigma} \in \mathcal{U}_{1}^{(n)} / U_{1}^{(n)}$ constituée des unités $b$ de $\widetilde{\mathfrak{M}}_{1}^{(n)}$ telles que $\phi(b) / b=\sigma\left(\varpi_{1}^{(n)}\right) / \varpi_{1}^{(n)}$ est une bijection vérifiant $v_{\sigma_{1} \sigma_{2}}=v_{\sigma_{1}} \sigma_{1}\left(v_{\sigma_{2}}\right)$ pour tous $\sigma_{1}, \sigma_{2} \in \mathfrak{g}_{1}^{(n)}$.

Notons-la $\digamma^{(n)}$; c'est l'application notée $\boldsymbol{N}_{M_{1}^{(n)} / K}$ dans [Fes01].

L'ensemble $\left\{\mathcal{U}_{1}^{(n)} / U_{1}^{(n)}\right\}_{n \in \mathbb{N}}$ est un système projectif de groupes pour les morphismes induits par les projections canoniques $\widetilde{N}_{M_{1}^{(n)} / M_{1}^{(m)}},(n \geqslant m \geqslant 0)$, et $\widetilde{N}_{M_{1}^{(n)} / M_{1}^{(0)}}\left(\mathcal{U}_{1}^{(n)} / U_{1}^{(n)}\right)=U(K)$. 


\section{UNE THÉORIE DU CORPS DE CLASSES LOCAL NON ABÉLIEN}

Soient $\mathcal{U}_{1}=\lim _{\longleftarrow} \mathcal{U}_{1}^{(n)}, U_{1}=\lim _{\longleftarrow} U_{1}^{(n)}$ et $\digamma=\lim _{\longleftarrow} \digamma^{(n)}$ (relativement aux projections canoniques), alors $\mathcal{U}_{1} / U_{1}=\lim _{\longleftarrow}\left(\mathcal{U}_{1}^{(n)} / U_{1}^{(n)}\right)$.

Proposition 8. Soit $\Phi$ le sous-groupe normal fermé de $G_{K}$ engendré par $\phi$. Il existe un groupe $\mathcal{U}_{1} / U_{1}$ construit récursivement à partir de $K$ et de $\phi$, sur lequel $G_{K} / \Phi$ opère canoniquement et une bijection $\digamma$ de $G_{K} / \Phi$ sur $\mathcal{U}_{1} / U_{1}$ telle que $\forall \sigma, \tau \in G_{K} / \Phi, \digamma(\sigma \tau)=\digamma(\sigma) \sigma \digamma(\tau)$.

En termes de séries formelles, $\operatorname{ker}\left(\Re_{1}^{(n)}\right)=\hat{\mathbb{Z}} \times \mathbb{F}_{q}[[X]]^{\times}$et $\mathfrak{G}_{1}^{(n)}(K, \phi) / \operatorname{ker}\left(\Re_{1}^{(n)}\right)$ s'identifie au groupe quotient du groupe

$$
\left\{\xi^{(n)} \in \widetilde{\mathbb{F}}_{q}[[X]]^{\times} ; \phi\left(\xi^{(n)}(X)\right) / \xi^{(n)}(X)=\left(\xi^{(n-1)}\right)_{0}(X) / X\right\}
$$

pour la loi :

$$
\xi^{(n)} \star \eta^{(n)}=\xi^{(n)} \cdot \eta^{(n)} \circ\left(\xi^{(n-1)}\right)_{0}
$$

par son sous-groupe $\mathbb{F}_{q}[[X]]^{\times}$et $\Re_{1}^{(n)}\left(\xi^{(n)}\right)=\left(\xi^{(n-1)}\right)_{0} ; \digamma^{(n)}$ s'identifie à la bijection réciproque de $\Re_{1}^{(n)}$.

\section{BIBLIOGRAPHIE}

Fes01 I. Fesenko, Nonabelian local reciprocity maps, in Class field theory - its centenary and prospect, ed. K. Miyake, Advanced Studies in Pure Mathematics, vol. 30 (Mathematical Society of Japan, Tokyo, 2001), 63-78.

Gur98 A. Gurevich, Description of Galois groups of local fields with the aid of power series, Dissertation, Humboldt University (1998).

HT01 M. Harris and R. Taylor, On the geometry and cohomology of some simple Shimura varieties, Annals of Mathematics Studies, vol. 151 (Princeton University Press, Princeton, NJ, 2001).

KdS96 H. Koch and E. de Shalit, Metabelian local class field theory, J. reine angew. Math. 478 (1996), $85-106$.

Lau88 F. Laubie, Extensions de Lie et groupes d'automorphismes de corps locaux, Compositio Math. 67 (1988), 165-189.

Neu94 J. Neukirch, Micro primes, Math. Ann. 298 (1994), 629-666.

Win83 J.-P. Wintenberger, Le corps des normes de certaines extensions infinies de corps locaux; applications, Ann. Sci. École Norm. Sup. (4) 16 (1983), 59-89.

Win78 J.-P. Wintenberger, Automorphismes et extensions galoisiennes de corps locaux, Thèse de $3^{\mathrm{e}}$ cycle, Institut Fourier, Université Scientifique et Médicale de Grenoble (1978).

Zin83 E.-W. Zink, Lokale projektive Klassenkörpertheorie. II, Math. Nachr. 114 (1983), 123-150.

François Laubie francois.laubie@unilim.fr

Xlim UMR 6172 CNRS - Université de Limoges, Département de Mathématiques, 123 avenue Albert Thomas, 87060 Limoges cedex, France 